\title{
Analytic Perturbation Theory and Renormalization Analysis of Matter Coupled to Quantized Radiation
}

\author{
Marcel Griesemer and David G. Hasler
}

\begin{abstract}
For a large class of quantum mechanical models of matter and radiation we develop an analytic perturbation theory for non-degenerate ground states. This theory is applicable, for example, to models of matter with static nuclei and non-relativistic electrons that are coupled to the UV-cutoff quantized radiation field in the dipole approximation. If the lowest point of the energy spectrum is a non-degenerate eigenvalue of the Hamiltonian, we show that this eigenvalue is an analytic function of the nuclear coordinates and of $\alpha^{3 / 2}, \alpha$ being the fine structure constant. A suitably chosen ground state vector depends analytically on $\alpha^{3 / 2}$ and it is twice continuously differentiable with respect to the nuclear coordinates.
\end{abstract}

\section{Introduction}

When a neutral atom or molecule made from static nuclei and non-relativistic electrons is coupled to the (UV-cutoff) quantized radiation field, the least point of the energy spectrum becomes embedded in the continuous spectrum due to the absence of a photon mass, but it remains an eigenvalue $[13,18]$. This ground state energy $E$ depends on the parameters of the system, such as the fine-structure constant, the positions of static nuclei, or, in the center of mass frame of a translation invariant model, the total momentum. The regularity of $E$ as a function of these parameters is of fundamental importance. For example, the accuracy of the Born-Oppenheimer approximation, a pillar of quantum chemistry, depends on the regularity of $E$ and on the regularity of the ground state projection as functions of the nuclear coordinates. If $E$ were an isolated eigenvalue, like it is in quantum mechanical description of molecules without radiation, then analyticity of $E$ with 
respect to any of the aforementioned parameters would follow from regular perturbation theory. But in QED the energy $E$ is not isolated and the analysis of its regularity is a difficult mathematical problem.

In the present paper we study the problem of regularity, described above, in a large class of models of matter and radiation where the Hamiltonian $H(s)$ depends analytically on complex parameters $s=\left(s_{1}, \ldots, s_{\nu}\right) \in \mathbb{C}^{\nu}$ and is defined for values of $s$ from a complex neighborhood of a compact set $K \subset \mathbb{R}^{\nu}$. Important properties of $H(s)$ are, that $H(\bar{s})=H(s)^{*}$ and that, for $s \in K$, the lowest point, $E(s)$, of the spectrum of $H(s)$ is a non-degenerate eigenvalue. Under further assumptions, described below, we show that $E(s)$ and the projection operator associated with the eigenspace of $E(s)$ are real-analytic functions of $s$ in a neighborhood of $K$. In particular, they are of class $C^{\infty}$ in this neighborhood. We apply this result to the Hamiltonian of a molecule with static nuclei and non-relativistic electrons that are coupled to the quantized radiation field in dipole approximation. By our choice of atomic units, this Hamiltonian depends on the fine-structure constant $\alpha$ only though a factor of $\alpha^{3 / 2}$ in front of the dipole interaction operator. Hence the role of the parameter $s$ may be played by $\alpha^{3 / 2}$ or, after a well-known unitary deformation argument [15], by the nuclear coordinates. The general theorem described above implies that the ground state energy, if it is a non-degenerate eigenvalue, depends analytically on $\alpha^{3 / 2}$ and the nuclear coordinates. The ground state projection is analytic in $\alpha^{3 / 2}$, and twice continuously differentiable with respect to the nuclear coordinates. This paper thus also gives an answer to the question about the presence of $\alpha$-dependent and logarithmically divergent coefficients in an expansion of the ground state energy in powers of $\alpha^{1 / 2}$ [4]: they do not occur when the dipole approximation is used.

A further consequence of our main result concerns the accuracy of the adiabatic approximation to the time evolution $U_{\tau}$ generated by the Schrödinger equation

$$
i \frac{d}{d t} \varphi_{t}=H(t / \tau) \varphi_{t}, \quad t \in[0, \tau],
$$

in the limit $\tau \rightarrow \infty$. If $H(s)$ satisfies the assumptions of our result mentioned above with $K=[0,1]$, then the ground state projection $P(s)$ is of class $C^{\infty}([0,1])$ and hence the adiabatic theorem without gap assumption implies that $\sup _{t \in[0, \tau]} \|(1-$ $P(t)) U_{\tau}(t) P(0) \|=o(1)$ as $\tau \rightarrow \infty[2,22]$. Previously, in all applications of the adiabatic theorem without gap assumption the differentiability of $P(s)$ was enforced or provided by the special form $H(s)=U(s) H U(s)^{-1}$ of $H(s)$ where $U(s)$ is a unitary and (strongly) differentiable operator $[1,2,21]$.

We now describe our main result in detail. We consider a class of Hamiltonians $H_{g}(s): D \subset \mathcal{H} \rightarrow \mathcal{H}$ depending on a parameter $s \in V$, where $V=\bar{V}$ is a complex neighborhood of some point $s_{0} \in \mathbb{R}^{\nu}$. For each $s \in V$,

$$
H_{g}(s)=H_{\mathrm{at}}(s) \otimes 1+1 \otimes H_{f}+g W(s),
$$

with respect to $\mathcal{H}=\mathcal{H}_{\text {at }} \otimes \mathcal{F}$, where $\mathcal{H}_{\text {at }}$ is an arbitrary complex Hilbert space and $\mathcal{F}$ denotes the symmetric Fock space over $L^{2}\left(\mathbb{R}^{3} \times\{1,2\}\right)$. We assume that 
$H_{\mathrm{at}}(\bar{s})=H_{\mathrm{at}}(s)^{*}$ for all $s \in V$ and that $\left(H_{\mathrm{at}}(s)\right)_{s \in V}$ is an analytic family of type (A). This means that the domain $D$ of $H_{\text {at }}(s)$ is independent of $s \in V$ and that $s \mapsto H_{\text {at }}(s) \varphi$ is analytic for all $\varphi \in D$. We assume, moreover, that $E_{\text {at }}\left(s_{0}\right)=$ $\inf \sigma\left(H_{\text {at }}\left(s_{0}\right)\right)$ is a simple and isolated eigenvalue of $H_{\text {at }}\left(s_{0}\right)$. The operator $H_{f}$ describes the energy of the bosons, and $g W(s)$ the interaction of the particle system described by $H_{\mathrm{at}}(s)$ and the bosons. In terms of creation and annihilation operators

$$
H_{f}=\sum_{\lambda=1,2} \int|\mathbf{k}| a_{\lambda}^{*}(\mathbf{k}) a_{\lambda}(\mathbf{k}) d^{3} \mathbf{k}
$$

and

$$
W(s)=\sum_{\lambda=1,2} \int G_{\bar{s}}(\mathbf{k}, \lambda)^{*} \otimes a(\mathbf{k}, \lambda)+G_{s}(\mathbf{k}, \lambda) \otimes a^{*}(\mathbf{k}, \lambda) d^{3} \mathbf{k},
$$

where, $(\mathbf{k}, \lambda) \mapsto G_{s}(\mathbf{k}, \lambda)$, for each $s \in V$ is an element of $L^{2}\left(\mathbb{R}^{3} \times\{1,2\}, \mathcal{L}\left(H_{\text {at }}\right)\right)$. We assume that $s \mapsto G_{s}$ is a bounded analytic function on $V$ and that

$$
\sup _{s \in V} \sum_{\lambda=1,2} \int\left\|G_{s}(\mathbf{k}, \lambda)\right\|^{2} \frac{1}{|\mathbf{k}|^{2+2 \mu}} d^{3} \mathbf{k}<\infty
$$

for some $\mu>0$. Based on these assumptions we show that a neighborhood $V_{0} \subset V$ of $s_{0}$ and a positive constant $g_{0}$ exist such that for $s \in V_{0}$ and all $g \in\left[0, g_{0}\right)$ the operator $H_{g}(s)$ has a non-degenerate eigenvalue $E_{g}(s)$ and a corresponding eigenvector $\psi_{g}(s)$ that are both analytic functions of $s \in V_{0}$. Moreover $E_{g}(s)=$ $\inf \sigma\left(H_{g}(s)\right)$ for $s \in \mathbb{R} \cap V$. Before commenting on the proof of this result we briefly review the literature.

In [4] the dependence on $\alpha$ of the ground state and the ground state energy, $E$, is studied for non-relativistic atoms that are minimally coupled to the quantized radiation field. It is shown that $E$ and a suitably chosen ground state vector have expansions in asymptotic series of powers of $\alpha$ with $\alpha$-dependent coefficients that may diverge logarithmically as $\alpha \rightarrow 0$. Smoothness is not expected and hence the dipole approximation seems necessary for our analyticity result. Much earlier, in [9], Fröhlich obtained results on the regularity of the ground state energy with respect to the total momentum $P$ for the system of a single quantum particle coupled linearly to a quantized field of massless scalar bosons. Let $H(P)$ denote the Hamiltonian describing this system at fixed total momentum $P \in \mathbb{R}^{3}$. The spectrum of $H(P)$ is of the form $[E(P), \infty)$ but $E(P)$ is not an eigenvalue for $P \neq 0$ [9] (see [14] for similar results on positive ions). For a non-relativistic particle of mass $M$, Fröhlich shows that $P \mapsto E(P)$ is differentiable a.e. in $\{|P|<$ $(\sqrt{3}-1) M\}$, and that $\nabla E(P)$ is locally Lipschitz [9, Lemma 3.1]. This work was recently and independently continued by Alessandro Pizzo and Thomas Chen for systems with a fixed ultraviolet cutoff $[7,19]$. After a unitary, $P$-dependent transformation of the Hamiltonian $H(P)$, Pizzo obtains a ground state vector $\phi^{\sigma}(P)$ that is Hölder continuous with respect to $P$ uniformly in an infrared cutoff $\sigma>0$. Chen studied the regularity of $E(P, \sigma)$ for a non-relativistic particle coupled minimally to the quantized radiation field [7]. He estimates $\left|\partial_{|p|}^{\beta}\left(E(|p|, \sigma)-p^{2} / 2\right)\right|$ 
uniformly in $\sigma>0$ for $\beta \leq 2$. He also asserts that $E(p, \sigma)$ is of class $C^{2}$ even for $\sigma=0$. In [15] Hunziker proves analyticity with respect to the nuclear coordinates for non-relativistic molecules without radiation. The ground state energy is isolated but the Hamiltonian is not analytic with respect the nuclear coordinates. It only becomes analytic after a suitable unitary deformation (see Section 3) introduced by Hunziker.

The results of the present paper are derived using the renormalization technique of Bach et al. [3,6], in a new version that we take from [10]. Like the authors of [10] we use a simplified renormalization map that consists of a Feshbach-Schur map and a scaling transformation only. In the corresponding spectral analysis the Hamiltonian is diagonalized, with respect to $H_{f}$, in a infinite sequence of renormalization steps. In each step the off-diagonal part becomes smaller, thanks to (1), and the spectral parameter is adjusted to enforce convergence of the diagonal part. This method provides a fairly explicit construction of an eigenvector of $H(s)$, even for complex $s$, where $H(s)$ is not self-adjoint. We show first, that the parameters of the renormalization analysis can be chosen independent of $s$ and $g$ in neighborhoods of $s=s_{0}$ and $g=0$, second, that all steps of the renormalization analysis preserve analyticity, and third, that all limits taken are uniform in $s$, which implies analyticity of the limit functions. On a technical level, these three points are the main achievements of this paper. The result that analyticity with respect to the spectral parameter is preserved under the renormalization map has been used in all previous papers working with the renormalization techniques of Bach et al. If asked about a proof, its authors would argue, that the renormalization map, as a map of operator kernels, is given by explicit formulas. A proof spelling out this argument, to our best knowledge, has never been published. Our proof of Proposition 17 , provides a simple alternative argument. The $\delta$-calculus introduced in [3] does not solve this problem.

It seems unlikely that another approach, not based on a renormalization analysis would yield a result similar to ours. The proof of analyticity requires the construction of an eigenvector for complex values of $s$ where $H(s)$ is not self-adjoint and hence, variational methods, for example, are not applicable. There is, of course, the tempting alternative approach to first introduce a positive photon mass $\sigma$ to separate the least energy from the rest of the spectrum. But the neighborhood of analyticity obtained in this way depends on the size of $\sigma$ and vanishes in the limit $\sigma \rightarrow 0$.

We conclude this introduction with a description of the organization of this paper along with the strategy of our proof.

In Section 2 we introduce the class of Hamiltonians $(H(s))_{s \in V}$, we formulate all hypotheses, and state the main results. In Section 3 they are applied to nonrelativistic QED in dipole approximation to prove our results mentioned above on the regularity with respect to $\alpha^{3 / 2}$ and the nuclear coordinates.

Section 4 describes the smoothed Feshbach transform $F_{\chi}(H)$ of an operator $H$ and the isomorphism $Q(H)$ between the kernels of $F_{\chi}(H)$ and $H$ (isospectrality 
of the Feshbach transform). The transform $H \mapsto F_{\chi}(H)$ was discovered in [3], and generalized to the form needed here in [12].

In Section 5 we perform a first Feshbach transformation on $H(s)-z$ to obtain an effective Hamiltonian $H^{(0)}[s, z]$ on $\mathcal{H}_{\text {red }}=P_{[0,1]}\left(H_{f}\right) \mathcal{F}, \mathcal{F}$ being the Fock-space. We show that $H^{(0)}(s, z)$ is analytic in $s$ and $z$. By the isospectrality of the Feshbach transform, the eigenvalue problem for $H(s)$ is now reduced to finding a value $z(s) \in \mathbb{C}$ such that $H^{(0)}(s, z(s))$ has a nontrivial kernel.

In Section 6 we introduce a Banach space $\mathcal{W}_{\xi}$ and a linear mapping $H$ : $\mathcal{W}_{\xi} \rightarrow \mathcal{L}\left(\mathcal{H}_{\text {red }}\right)$. The renormalization transformation $\mathcal{R}_{\rho}$ is defined on a polydisc $\mathcal{B}(\rho / 2, \rho / 8, \rho / 8) \subset H\left(\mathcal{W}_{\xi}\right)$ as the composition of a Feshbach transform and a rescaling $\mathbf{k} \mapsto \rho \mathbf{k}$ of the photon momenta $\mathbf{k} . \rho \in(0,1)$ is the factor by which the energy scale is reduced in each renormalization step. $\mathcal{R}_{\rho}$ takes values in $\mathcal{L}\left(\mathcal{H}_{\text {red }}\right)$ and, like the Feshbach transform, it is isospectral.

In Section 7 it is shown that the analyticity of a family of Hamiltonians is preserved under the renormalization transformation. This is one of the key properties on which our strategy is based.

Sections 8 and 9 are devoted to the solution of our spectral problem for $H^{(0)}(s, z)$ by iterating the Renormalization map. Since this procedure is pointwise in $s$ with estimates that hold uniformly on $V$, we drop the parameter $s$ for notational simplicity.

In Sections 8 we define $H^{(n)}[s, z]=\mathcal{R}^{n} H^{(0)}[s, z]$ for values of the spectral parameter $z$ from non-empty sets $U_{n}(s)$. These sets are nested, $U_{n}(s) \supset U_{n+1}(s)$, and they shrink to a point, $\cap_{n} U_{n}(s)=\left\{z_{\infty}(s)\right\}$. Since $H^{(n)}\left(z_{\infty}\right) \rightarrow$ const $H_{f}$ as $n \rightarrow \infty$ in the norm of $\mathcal{L}\left(\mathcal{H}_{\text {red }}\right)$ and since the vacuum $\Omega$ is an eigenvector $H_{f}$ with eigenvalue zero, it follows, by the isospectrality of $\mathcal{R}$, that zero is an eigenvalue of $H^{(n)}\left(z_{\infty}\right)$ for all $n$.

In Section 9 a vector $\varphi_{n}$ in the kernel of $H^{(n)}\left(z_{\infty}\right)$ is computed by compositions of scaling transformations and mappings $Q\left(H^{(k)}\left(z_{\infty}\right)\right), k \geq n$, applied to $\Omega$. $\varphi_{g s}=Q\left(H^{(0)}\left(z_{\infty}\right)\right) \varphi_{0}$ is an eigenvector of $H$ with eigenvalue $z_{\infty}$.

In Section 10 we show that $s \mapsto z_{\infty}(s)$ is analytic and that $Q\left(H^{(n)}\left(z_{\infty}(s)\right)\right)$ maps analytic vectors to analytic vectors. Since the vacuum $\Omega$ is trivially analytic in $s$, it follows that $\varphi_{g s}(s)$ is analytic in $s$.

In the Appendices A and B we collect technical auxiliaries and for completeness we give a proof of $H^{(n)}\left(z_{\infty}\right) \rightarrow$ const $H_{f}$ as $n \rightarrow \infty$, although this property is not used explicitly.

\section{Assumptions and main results}

We consider families of (unbounded) closed operators $H_{g}(s): D\left(H_{g}(s)\right) \subset \mathcal{H} \rightarrow \mathcal{H}$, $s \in V$, where $V \subset \mathbb{C}^{\nu}$ is open, symmetric with respect to complex conjugation and $V \cap \mathbb{R}^{\nu} \neq \emptyset$. The Hilbert space $\mathcal{H}$ is a tensor product

$$
\mathcal{H}=\mathcal{H}_{\mathrm{at}} \otimes \mathcal{F}, \quad \mathcal{F}=\bigoplus_{n=0}^{\infty} S_{n}\left(\otimes^{n} \mathfrak{h}\right),
$$


of an arbitrary, separable, complex Hilbert space $\mathcal{H}_{\text {at }}$ and the symmetric Fock space $\mathcal{F}$ over the Hilbert space $\mathfrak{h}:=L^{2}\left(\mathbb{R}^{3} \times\{1,2\} ; \mathbb{C}\right)$ with norm given by

$$
\|h\|^{2}:=\sum_{\lambda=1,2} \int|h(\mathbf{k}, \lambda)|^{2} d^{3} \mathbf{k}, \quad h \in \mathfrak{h} .
$$

Here $S_{0}\left(\otimes^{0} \mathfrak{h}\right):=\mathbb{C}$ and for $n \geq 1, S_{n} \in \mathcal{L}\left(\otimes^{n} \mathfrak{h}\right)$ denotes the orthogonal projection onto the subspace left invariant by all permutation of the $n$ factors of $\mathfrak{h}$. To simplify our notation we set

$$
k:=(\mathbf{k}, \lambda), \quad \int d k:=\sum_{\lambda=1,2} \int d^{3} \mathbf{k}, \quad|k|:=|\mathbf{k}|,
$$

throughout the rest of this paper.

For each $s \in V$, the operator $H_{g}(s)$ is a sum

$$
H_{g}(s)=H_{\mathrm{at}}(s) \otimes 1+1 \otimes H_{f}+g W(s),
$$

of a closed operator $H_{\text {at }}(s)$ in $\mathcal{H}_{\text {at }}$, the second quantization, $H_{f}$, of the operator $\omega$ on $L^{2}\left(\mathbb{R}^{3} \times\{1,2\}\right)$ of multiplication with

$$
\omega(k)=|k|,
$$

and an interaction operator $g W(s), g \geq 0$ being a coupling constant. The operator $W(s)$ is the sum

$$
W(s)=a\left(G_{\bar{s}}\right)+a^{*}\left(G_{s}\right)
$$

of an annihilation operator, $a\left(G_{\bar{s}}\right)$, and a creation operator, $a^{*}\left(G_{s}\right)$, associated with an operator $G_{s} \in \mathcal{L}\left(\mathcal{H}_{\text {at }}, \mathcal{H}_{\text {at }} \otimes \mathfrak{h}\right)$. The creation operator, $a^{*}\left(G_{s}\right)$, as usual, is defined as the closure of the linear operator in $\mathcal{H}$ given by

$$
a^{*}\left(G_{s}\right)(\varphi \otimes \psi):=\sqrt{n} S_{n}\left(G_{s} \varphi \otimes \psi\right),
$$

if $\varphi \in \mathcal{H}_{\text {at }}$ and $\psi \in S_{n-1}\left(\otimes^{n-1} \mathfrak{h}\right)$. The annihilation operator $a\left(G_{s}\right)$ is the adjoint of $a^{*}\left(G_{s}\right)$.

Hypotheses I below will imply that $H_{g}(s)$ is well defined on $D\left(H_{\mathrm{at}}(s)\right) \otimes$ $D\left(H_{f}\right)$ and closable. To formulate it, some preliminary remarks are necessary. Let $L^{2}\left(\mathbb{R}^{3}, \mathcal{L}\left(\mathcal{H}_{\mathrm{at}}\right)\right)$ be the Banach space of (weakly) measurable and square integrable functions from $\mathbb{R}^{3}$ to $\mathcal{L}\left(\mathcal{H}_{\text {at }}\right)$. Every element $T$ of this space defines a linear operator $T: \mathcal{H}_{\mathrm{at}} \rightarrow L^{2}\left(\mathbb{R}^{3}, \mathcal{H}_{\mathrm{at}}\right)$ by

$$
(T \varphi)(k):=T(k) \varphi .
$$

This operator is bounded and $\|T\| \leq\|T\|_{2}$. Since $L^{2}\left(\mathbb{R}^{3}, \mathcal{H}_{\mathrm{at}}\right) \simeq \mathcal{H}_{\mathrm{at}} \otimes \mathfrak{h}$, we may consider $T$ as an element of $\mathcal{L}\left(\mathcal{H}_{\text {at }}, \mathcal{H}_{\text {at }} \otimes \mathfrak{h}\right)$ and hence $L^{2}\left(\mathbb{R}^{3}, \mathcal{L}\left(\mathcal{H}_{\text {at }}\right)\right)$ as a subspace embedded in $\mathcal{L}\left(\mathcal{H}_{\text {at }}, \mathcal{H}_{\text {at }} \otimes \mathfrak{h}\right)$.

Hypothesis I. The mapping $s \mapsto G_{s}$ is an bounded analytic function on $V$ with values in $L^{2}\left(\mathbb{R}^{3}, \mathcal{L}\left(\mathcal{H}_{\mathrm{at}}\right)\right)$, and there exists a $\mu>0$, such that

$$
\sup _{s \in V} \int \frac{1}{|k|^{2+2 \mu}}\left\|G_{s}(k)\right\|^{2} d k<\infty .
$$


By Lemma 25, $\left\|a^{\#}\left(G_{s}\right)\left(H_{f}+1\right)^{-1 / 2}\right\| \leq\left\|G_{s}\right\|_{\omega}$, where

$$
\left\|G_{s}\right\|_{\omega}^{2}:=\int_{\mathbb{R}^{3}}\left\|G_{s}(k)\right\|^{2}\left(|k|^{-1}+1\right) d k .
$$

Hence Hypothesis I implies that $W(s)$ and $W(s)^{*}$ are well defined on $\mathcal{H}_{\text {at }} \otimes D\left(H_{f}\right)$. It follows that $H_{g}(s)$ is defined on $D\left(H_{\text {at }}(s)\right) \otimes D\left(H_{f}\right)$ and that the adjoint of this operator has a domain which contains $D\left(H_{\text {at }}(s)\right) \otimes D\left(H_{f}\right)$. This subspace is dense because $H_{\text {at }}(s)$ is closed. That is, $H_{g}(s): D\left(H_{\text {at }}(s)\right) \otimes D\left(H_{f}\right) \subset \mathcal{H} \rightarrow \mathcal{H}$ has a densely defined adjoint, and hence it is closable.

\section{Hypothesis II.}

(i) $H_{\text {at }}(s)$ is an analytic family of operators in the sense of Kato and $H_{\text {at }}(s)^{*}=$ $H_{\text {at }}(\bar{s})$ for all $s \in V$. In particular, $H_{\text {at }}(s)$ is self-adjoint for $s \in \mathbb{R}^{\nu} \cap V$.

(ii) There exists a point $s_{0} \in V \cap \mathbb{R}^{\nu}$ such that $E_{\text {at }}\left(s_{0}\right):=\inf \sigma\left(H_{\text {at }}\left(s_{0}\right)\right)$ is an isolated, non-degenerate eigenvalue of $H_{\mathrm{at}}\left(s_{0}\right)$.

For the notion of an analytic family of operators in the sense of Kato we refer to [20]. The definition given there readily generalizes to several complex variables. We recall that a function of several complex variables is called analytic if it is analytic in each variable separately.

By Hypothesis II, (ii), and the Kato-Rellich theorem of analytic perturbation theory [20], there is exactly one point $E_{\text {at }}(s)$ of $\sigma\left(H_{\text {at }}(s)\right)$ near $E_{\text {at }}\left(s_{0}\right)$, for $s$ near $s_{0}$, and this point is a non-degenerate eigenvalue of $H_{\text {at }}(s)$. Moreover, for $s$ near $s_{0}$, there is an analytic projection onto the eigenvector of $E_{\text {at }}(s)$, which is given by

$$
P_{\mathrm{at}}(s):=\frac{1}{2 \pi i} \int_{\left|E_{\mathrm{at}}(s)-z\right|=\epsilon}\left(z-H_{\mathrm{at}}(s)\right)^{-1} d z,
$$

for $\epsilon>0$ sufficiently small. We set $\bar{P}_{\text {at }}(s)=1-P_{\text {at }}(s)$.

Hypothesis III. Hypothesis II holds and there exists a neighborhood $\mathcal{U} \subset V \times \mathbb{C}$ of $\left(s_{0}, E_{\text {at }}\left(s_{0}\right)\right)$ such that for all $(s, z) \in \mathcal{U},\left|E_{\text {at }}(s)-z\right|<1 / 2$ and

$$
\sup _{(s, z) \in \mathcal{U}} \sup _{q \geq 0}\left\|\frac{q+1}{H_{\text {at }}(s)-z+q} \bar{P}_{\text {at }}(s)\right\|<\infty .
$$

Remarks.

1. Hypothesis III is satisfied, e.g., if Hypothesis II holds and $H_{\text {at }}(s)$ is an analytic family of type (A), see Corollary 3 .

2. The condition $\left|E_{\text {at }}(s)-z\right|<1 / 2$ is needed in the proof of Theorem 13 and related to the constant $3 / 4$ in the construction of $\chi$. Since $s \mapsto E_{\text {at }}(s)$ is continuous, it can always be met by choosing $\mathcal{U}$ sufficiently small. However, the smaller we choose $\mathcal{U}$ the smaller we will have to choose the coupling constant $g$. Optimal bounds on $g$ could possibly be obtained by scaling the operator such that the gap in $H_{\text {at }}$ is comparable to one.

We are now ready to state the main results. 
Theorem 1. Suppose Hypotheses I, II and III hold. Then there exists a neighborhood $V_{0} \subset V$ of $s_{0}$ and a positive constant $g_{0}$ such that for all $s \in V_{0}$ and all $g<g_{0}$ the operator $H_{g}(s)$ has an eigenvalue $E_{g}(s)$ and a corresponding eigenvector $\psi_{g}(s)$ that are both analytic functions of $s \in V_{0}$ such that

$$
E_{g}(s)=\inf \sigma\left(H_{g}(s)\right)
$$

for $s \in V_{0} \cap \mathbb{R}^{\nu}$.

Remark. For $s \in V_{0} \cap \mathbb{R}^{\nu}$ and $g$ sufficiently small, the eigenvalue $E_{g}(s)$ is nondegenerate by Hypothesis II (ii) and a simple overlap estimate [5].

Corollary 2. Assume Hypotheses I and II are satisfied and that there exists a $C$ such that for all $s \in V$

$$
\operatorname{Re}\left\langle\varphi, H_{\mathrm{at}}(s) \varphi\right\rangle \geq-C\langle\varphi, \varphi\rangle, \quad \text { for } \varphi \in D\left(H_{\mathrm{at}}(s)\right) .
$$

Then the conclusions of Theorem 1 hold.

Proof. It suffices to verify Hypothesis III, then the corollary will follow from Theorem 1. For all $s \in V, z \in \mathbb{C}$ with $\left|z-E_{\text {at }}\left(s_{0}\right)\right| \leq 1, q \geq q^{*}:=C+\left|E_{\text {at }}\left(s_{0}\right)\right|+2$, and $\varphi \in D\left(H_{\text {at }}(s)\right)$ with $\|\varphi\|=1$,

$$
\begin{aligned}
\left\|\left(H_{\mathrm{at}}(s)-z+q\right) \varphi\right\| & \geq \operatorname{Re}\left\langle\varphi,\left(H_{\mathrm{at}}(s)-z+q\right) \varphi\right\rangle \\
& \geq q-C-\left|E_{\mathrm{at}}\left(s_{0}\right)\right|-1 \geq 1 .
\end{aligned}
$$

Since $H_{\mathrm{at}}(s)^{*}=H_{\mathrm{at}}(\bar{s})$ an analog estimate holds for $H_{\mathrm{at}}(s)^{*}$. This proves that $B_{1}\left(E_{\text {at }}\left(s_{0}\right)\right) \subset \rho\left(H_{\text {at }}(s)+q\right)$ for $s \in V, q \geq q^{*}$, and that

$$
\sup _{s \in V,\left|z-E_{\text {at }}\left(s_{0}\right)\right| \leq 1, q \geq q^{*}}\left\|\frac{q+1}{H_{\text {at }}(s)-z+q}\right\| \leq \frac{q^{*}}{q^{*}-C+\left|E_{\text {at }}\left(s_{0}\right)\right|+1} .
$$

We now turn to the case where $0 \leq q \leq q^{*}$. The set

$$
\Gamma:=\left\{(s, z) \in \mathbb{C}^{\nu} \times \mathbb{C} \mid z \in \rho\left(H(s) \uparrow \bar{P}_{\text {at }}(s) \mathcal{H}\right)\right\}
$$

is open and $\left(H_{\mathrm{at}}(s)-z\right)^{-1} \bar{P}_{\mathrm{at}}(s)$ is analytic on $\Gamma[20]$. On the other hand

$$
\gamma:=\left\{\left(s_{0}, E_{\text {at }}\left(s_{0}\right)-q\right) \mid 0 \leq q \leq q^{*}\right\}
$$

is a compact subset of $\Gamma$. It follows that the distance between $\gamma$ and the complement of $\Gamma$ is positive. Thus if $g$ and $\delta>0$ are small enough, then

$$
\left\{(s, z-q):\left|s-s_{0}\right| \leq \delta,\left|z-E_{\text {at }}\left(s_{0}\right)\right| \leq \delta, 0 \leq q \leq q^{*}\right\}
$$

is a compact subset of $\Gamma$ on which $(H(s)-z)^{-1} \bar{P}_{\text {at }}(s)$ is uniformly bounded. Comparing with (4) we conclude that for $\delta<1$ so small that $B_{\delta}\left(s_{0}\right) \subset V$ the Hypothesis III holds with $U=B_{\delta}\left(s_{0}\right) \times B_{\delta}\left(E\left(s_{0}\right)\right)$

The following corollaries prove the assertions in the introduction. 
Corollary 3. Suppose Hypothesis I holds and let $H_{\mathrm{at}}(s)$ be an analytic family of type (A) with $H_{\mathrm{at}}(s)^{*}=H_{\mathrm{at}}(\bar{s})$ for all $s \in V$. If $E\left(s_{0}\right)=\inf \sigma\left(H\left(s_{0}\right)\right)$ is a nondegenerate isolated eigenvalue of $H_{\text {at }}\left(s_{0}\right)$, then the conclusions of Theorem 1 hold.

Proof. By Corollary 2 it suffices to show that (3) holds. To this end we set

$$
T(s):=H_{\text {at }}(s)-E_{\text {at }}\left(s_{0}\right)
$$

and $R:=\left(T\left(s_{0}\right)+1\right)^{-1}$. Since $T(s)$ is an analytic family of type $(\mathrm{A})$, the operators $T(s) R$ and $R T(s)$ are bounded and weakly analytic, hence strongly analytic [16]. It follows that $\left(T(s)-T\left(s_{0}\right)\right) R \rightarrow 0$ and $R\left(T(s)-T\left(s_{0}\right)\right) \rightarrow 0$ as $s \rightarrow 0$. By abstract interpolation theory

$$
R^{1 / 2}\left(T(s)-T\left(s_{0}\right)\right) R^{1 / 2} \rightarrow 0 \quad s \rightarrow s_{0} .
$$

We choose $\varepsilon>0$ so that $B_{\varepsilon}\left(s_{0}\right) \subset V$ and

$$
\sup _{|s|<\varepsilon}\left\|R^{1 / 2}\left(T(s)-T\left(s_{0}\right)\right) R^{1 / 2}\right\| \leq 1 / 2 .
$$

It follows that $\left|\left\langle\varphi,\left[T(s)-T\left(s_{0}\right)\right] \varphi\right\rangle\right| \leq(1 / 2)\left\langle\varphi,\left(T\left(s_{0}\right)+1\right) \varphi\right\rangle$ and hence that

$$
\begin{aligned}
\operatorname{Re}\langle\varphi, T(s) \varphi\rangle & \geq\left\langle\varphi, T\left(s_{0}\right) \varphi\right\rangle-\left|\left\langle\varphi,\left[T(s)-T\left(s_{0}\right)\right] \varphi\right\rangle\right| \\
& \geq \frac{1}{2}\left\langle\varphi, T\left(s_{0}\right) \varphi\right\rangle-\frac{1}{2}\langle\varphi, \varphi\rangle \geq-\frac{1}{2}\langle\varphi, \varphi\rangle
\end{aligned}
$$

which proves (3).

Remark. Embedded eigenvalues generically disappear under perturbations. However, if a non-degenerate eigenvalue persists, one might conjecture that the eigenvalue and a suitable eigenvector are analytic functions of the perturbation parameter, provided the Hamiltonian is analytic in this parameter. This conjecture is wrong, as the following example shows. Let $\mathcal{H}=L^{2}(\mathbb{R}) \oplus\left(\mathbb{C} \oplus L^{2}(\mathbb{R})\right)$ and let

$$
H(s)=H_{1}(s) \oplus H_{2}(s)
$$

where

$$
H_{1}(s):=\left(-\frac{d^{2}}{d x^{2}}-s^{2} \chi_{[-1,1]}\right), \quad \text { in } \quad L^{2}(\mathbb{R}),
$$

and where $H_{2}(s)$ is defined as follows. We choose $\eta \in L^{2}(\mathbb{R})$ as $\eta(x)=e^{-x^{2}}$ and we use $|x|$ to denote the operator of multiplication by the function $x \mapsto|x|$ in $L^{2}(\mathbb{R})$. Then

$$
H_{2}(s):=\left(\begin{array}{ll}
0 & s\langle\eta, \cdot\rangle \\
s \eta & |x|
\end{array}\right), \quad \text { in } \quad \mathbb{C} \oplus L^{2}(\mathbb{R}),
$$

The operator $H_{1}(s)$ has no eigenvalues for $s=0$, and for $s \in \mathbb{R}$ near $s=0$ the lowest point of its spectrum is the only eigenvalue and it is non-degenerate. The operator $H_{2}(s)$ has the simple eigenvalue 0 if $s=0$ and it is not hard to see that it has no eigenvalues for $s \neq 0$. Therefore, for $s \in \mathbb{R}$ near $s=0$,

$$
E(s):= \begin{cases}\inf \sigma\left(H_{1}(s)\right), & s \neq 0, \\ 0, & s=0\end{cases}
$$


is the only eigenvalue of $H(s)$, it is simple and continuous in $s$. Yet there is no choice of an eigenvector depending continuously on $s$ in spite of the fact that the operators $H(s)$ for $s \in \mathbb{C}$ form an analytic family of type (A).

Corollary 4. Suppose the assumptions of Theorem 1, Corollary 2 or Corollary 3 are satisfied for all $s_{0}$ of a compact set $K \subset V \cap \mathbb{R}^{\nu}$. Then there exists a neighborhood $V_{0} \subset V$ of $K$ and a positive $g_{0}$ such that for all $s \in V_{0}$ and all $g<g_{0}$ there is an analytic complex-valued function $E_{g}$ and an analytic projection-valued functions $P_{g}$ on $V_{0}$, such that

$$
H_{g}(s) P_{g}(s)=E_{g}(s) P_{g}(s), \quad \text { for } \quad s \in V_{0},
$$

and $E_{g}(s)=\inf \sigma\left(H_{g}(s)\right)$ for $s \in V_{0} \cap \mathbb{R}^{\nu}$.

Proof. By the compactness of $K$ there exist open sets $V_{1} \ldots, V_{N} \subset \mathbb{C}^{\nu}$ and positive numbers $g_{1}, \ldots, g_{N}$ provided by Theorem 1 , such that

$$
K \subset \bigcup_{j=1}^{N} V_{j} .
$$

Let $E_{j}(s)$ and $\psi_{j}(s)$ be the corresponding eigenvalues and eigenvectors defined for $s \in V_{j}$ and $g<g_{j}$. We may assume that $\left\langle\psi_{j}(\bar{s}), \psi_{j}(s)\right\rangle \neq 0$ for all $s \in V_{j}$ and all $j$. Then the operators $P_{j}(s): \mathcal{H} \rightarrow \mathcal{H}$ defined by

$$
P_{j}(s) \varphi=\frac{\left\langle\psi_{j}(\bar{s}), \varphi\right\rangle}{\left\langle\psi_{j}(\bar{s}), \psi_{j}(s)\right\rangle} \psi_{j}(s)
$$

are analytic functions of $s \in V_{j}, P_{j}(s)^{2}=P_{j}(s)$, and $H_{g}(s) P_{j}(s)=E_{j}(s) P_{j}(s)$. We choose $g_{0} \leq \min \left\{g_{1}, \ldots, g_{N}\right\}$ so small, that all eigenvalues $E_{1}(s), \ldots, E_{N}(s)$ are non-degenerate for real $s$ and $g \in\left[0, g_{0}\right)$, and we define $V_{0}:=\cup_{j=1}^{N} V_{j}$. Then for $g<g_{0}$ and $s \in V_{i} \cap V_{j} \cap \mathbb{R}^{\nu}$,

$$
E_{i}(s)=E_{j}(s), \quad P_{i}(s)=P_{j}(s),
$$

and hence, by analyticity, (5) must hold for all $s \in V_{i} \cap V_{j}$. This proves that $E_{g}(s)$ and $P_{g}(s)$ are well-defined on $V_{0}$ by $E_{g}(s):=E_{j}(s)$ and $P_{g}(s):=P_{j}(s)$ for $s \in V_{j}$, and have the desired properties.

\section{Non-relativistic QED in dipole-approximation}

In this section we apply Theorem 1 to the Hamiltonians describing molecules made from static nuclei and non-relativistic electrons coupled to the UV-cutoff quantized radiation field in dipole approximation. For justifications of this model see $[8,11]$.

A (pure) state of system of $N$ spinless electrons and transversal photons is described by a vector in the Hilbert space $\mathcal{H}=L_{a}\left(\mathbb{R}^{3 N} ; \mathbb{C}\right) \otimes \mathcal{F}$, where $L_{a}^{2}\left(\mathbb{R}^{3 N} ; \mathbb{C}\right)$ denotes the space of square integrable, antisymmetric functions of $\left(x_{1}, \ldots, x_{N}\right) \in$ $\mathbb{R}^{3 N}$, and $\mathcal{F}$ is the symmetric Fock space over $L^{2}\left(\mathbb{R}^{3} \times\{1,2\}\right)$. We choose units where $\hbar, c$, and four times the Rydberg energy are equal to one, and we express 
all positions in multiples of one half of the Bohr-radius, which, in our units, agrees with the fine-structure constant $\alpha$. In these units the Hamiltonian reads

$$
H(X, \alpha)=H_{\mathrm{at}}(X)+\alpha^{3 / 2} \sum_{j=1}^{N} g\left(x_{j}\right) x_{j} \cdot E(0)+H_{f},
$$

with

$$
H_{\mathrm{at}}(X):=\sum_{j=1}^{N}\left(-\Delta_{j}\right)+\sum_{j<l} \frac{1}{\left|x_{l}-x_{j}\right|}-\sum_{j, k} \frac{Z_{j}}{\left|x_{j}-X_{k}\right|}
$$

where $Z_{1}, \ldots, Z_{K} \in \mathbb{N}$ denote atomic numbers, and

$$
E(0)=\sum_{\lambda=1,2} \int_{|\mathbf{k}|<\Lambda} d \mathbf{k} \sqrt{|\mathbf{k}|} i \varepsilon(\mathbf{k}, \lambda)\left(a^{*}(\mathbf{k}, \lambda)-a(\mathbf{k}, \lambda)\right),
$$

is the quantized electric field evaluated at the origin $0 \in \mathbb{R}^{3}$. The ultraviolet cutoff $\Lambda>0$ is an arbitrary but finite constant, the polarization vectors $\varepsilon(\mathbf{k}, 1)$ and $\varepsilon(\mathbf{k}, 2)$ are unit vectors in $\mathbb{R}^{3}$ that are orthogonal to each other and to $\mathbf{k}$, and $g \in C_{0}^{\infty}\left(\mathbb{R}^{3}\right)$ is a space-cutoff with $g \equiv 1$ on an open ball $B \subset \mathbb{R}^{3}$ containing the positions $X_{1}, \ldots, X_{K}$ of the nuclei. The following theorem is a consequence of Corollary 3 .

Theorem 5. Suppose inf $\sigma\left(H_{\mathrm{at}}(X)\right)$ is a non-degenerate and isolated eigenvalue of $H_{\mathrm{at}}(X)$. Then in a neighborhood of $\alpha=0$ the ground state energy and a suitably chosen ground state vector are real-analytic functions of $\alpha^{3 / 2}$.

Proof. For $s \in V:=B_{1}(0)$ we define $H_{g}(s)$ by the operator (2) with

$$
G_{s}(\mathbf{k}, \lambda):=s \sum_{j=1}^{N} \sqrt{|\mathbf{k}|} \chi(|\mathbf{k}| \leq \Lambda) i \varepsilon(\mathbf{k}, \lambda) \cdot x_{j} g\left(x_{j}\right)
$$

so that $H_{g}\left(\alpha^{3 / 2} / g\right)=H(X, \alpha)$. The Hamiltonian $H_{\mathrm{at}}(X)$ is trivially analytic of type $(A)$ in $V$ and $G_{s}$ satisfies Hypothesis I with, for example, $\mu=1 / 2$. Hence the conclusions of Theorem 1 holds by Corollary 3 . This proves the theorem.

The next theorem concerns the regularity of inf $\sigma(H(X, \alpha))$ with respect to the nuclear coordinates $X=\left(X_{1}, \ldots, X_{K}\right) \in \mathbb{R}^{3 K}$.

Theorem 6. Suppose inf $\sigma\left(H_{\mathrm{at}}(X)\right)$ is a non-degenerate and isolated eigenvalue of $H_{\mathrm{at}}(X)$, where $X \in B^{K}$ and $X_{r} \neq X_{s}$ for $r \neq s$. Then for $\alpha$ sufficiently small, there exists a neighborhood $U$ of $0 \in \mathbb{R}^{3 K}$ such that:

(a) For each $\xi \in U, E(\xi)=\inf \sigma(H(X+\xi))$ is an eigenvalue of $H(X+\xi)$ and a real-analytic function of $\xi$.

(b) There is an eigenvector belonging to $E(\xi)$, which is of class $C^{2}$ with respect to $\xi$. 
Remark. The operators $H_{\text {at }}(X)$ do not form an analytic family in the sense of Kato and hence Theorem 1 is not immediately applicable. This problem is circumvented by a well-known deformation argument [15].

Proof. By assumption on $X_{1}, \ldots, X_{K}$, we can find functions $f_{1}, \ldots, f_{K} \in C_{0}^{\infty}\left(\mathbb{R}^{3}\right)$, with $\operatorname{supp}\left(f_{r}\right) \subset B$ and $f_{r}\left(X_{s}\right)=\delta_{r s}$. For each $\xi=\left(\xi_{1}, \ldots, \xi_{K}\right) \in \mathbb{R}^{3 K}$ we define a vector field $v_{\xi}$ on $\mathbb{R}^{3}$, by

$$
v_{\xi}(x)=\sum_{r=1}^{K} \xi_{r} f_{r}(x) .
$$

It is not hard to see that for small $\xi$ the map

$$
\phi_{\xi}:\left(x_{1}, \ldots, x_{N}\right) \mapsto\left(x_{1}+v_{\xi}\left(x_{1}\right), \ldots, x_{N}+v_{\xi}\left(x_{N}\right)\right)
$$

is a diffeomorphism of $\mathbb{R}^{3 N}[15]$. Moreover,

$$
U_{\xi} \psi:=\left|D \phi_{\xi}\right|^{1 / 2}\left(\psi \circ \phi_{\xi}\right) \text {, }
$$

defines a unitary transformation $U_{\xi}$ on $\mathcal{H}$. A straightforward calculation shows that, for real and small $\xi$,

$$
\widetilde{H}(\xi):=U_{\xi} H(X+\xi) U_{\xi}^{-1}=H_{\mathrm{at}}(X ; \xi)+W(\xi)+H_{f},
$$

with

$$
\begin{gathered}
W(\xi)=\alpha^{3 / 2} \sum_{j=1}^{N} g\left(x_{j}\right)\left(x_{j}+v_{\xi}\left(x_{j}\right)\right) \cdot E(0) \\
H_{\mathrm{at}}(X ; \xi)=T_{\xi}-\sum_{r, j} Z_{k} V_{\xi}\left(x_{j}, X_{r}\right)+\sum_{j<l} V_{\xi}\left(x_{j}, x_{l}\right)
\end{gathered}
$$

where

$$
\begin{aligned}
T_{\xi} & =U_{\xi} \sum_{j=1}^{N}\left(-\Delta_{j}\right) U_{\xi}^{-1} \\
V_{\xi}(x, y) & =\left|x-y+v_{\xi}(x)-v_{\xi}(y)\right|^{-1} .
\end{aligned}
$$

In (6) we used that $g\left(x+v_{\xi}(x)\right)=g(x)$, by the smallness of $\xi$. In [15] it is proven using standard estimates that $H_{\text {at }}(X, \xi)$ has an extension to $\xi \in \mathbb{C}^{3 K}$ and this extension is an analytic family of type $(\mathrm{A})$ for $\xi$ in a neighborhood of zero. One easily verifies that $W(\xi)$ satisfies Hypothesis I. It follows that Corollary 3 is applicable and thus, for small $\alpha, \widetilde{H}(\xi)$ has an eigenvalue $E(\xi)$ with eigenvector $\varphi_{\xi}$, both analytic in $\xi$, and $\varphi_{\xi}$ is a ground state for real $\xi$. Since, for small and real $\xi$, $H(\xi+X)$ is unitarily equivalent to $\widetilde{H}(\xi)$, (a) follows.

To prove (b) we show that $\xi \mapsto U_{\xi}^{-1} \varphi_{\xi}$ is a $C^{2}$ function in a neighborhood of zero. Let $S_{\xi}:=U_{\xi}^{-1}$. Throughout the proof, with the exception of Step 3, we assume that $\xi$ is real. Using dominated convergence, one sees that $\xi \mapsto S_{\xi} \varphi$ is continuous for $\varphi \in \mathcal{H} \cap\left(C_{0}^{\infty}\left(\mathbb{R}^{3 N}\right) \otimes \mathcal{F}\right)$. Since such functions constitute a dense subset of $\mathcal{H}$ and $S_{\xi}$ is uniformly bounded, it follows that $\xi \mapsto S_{\xi}$ is strongly 
continuous. We shall adopt the following conventions in this proof: the labels $\alpha, \beta$ run over the set $\{1, \ldots, K\} \times\{1,2,3\}$, and $\partial_{\beta}=\partial / \partial \xi_{\beta}$ with $\xi_{\beta}=\left(\xi_{j}\right)_{s}$ for $\beta=(j, s)$; the labels $\mu, \nu$ run over the set $\{1, \ldots, N\} \times\{1,2,3\}$ and $p_{\mu}=\left(p_{l}\right)_{s}$ for $\mu=(l, s)$.

Step 1: If $\psi \in D(|p|)$, then $\xi \mapsto S_{\xi} \psi$ is $C^{1}$ and for all $\beta, \partial_{\beta} S_{\xi} \psi=S_{\xi} A_{\beta}(\xi) \psi$ with

$$
\begin{aligned}
{\left[A_{\beta}(\xi) \psi\right](x)=} & \left.\left|D \phi_{\xi}(x)\right|^{1 / 2} \frac{d}{d s}\left|D \phi_{\xi+s e_{\beta}}^{-1}\left(\phi_{\xi}(x)\right)\right|^{1 / 2}\right|_{s=0} \psi(x) \\
& +\left.\sum_{j=1}^{N} \frac{d}{d s} \phi_{\xi+s e_{\beta}}^{-1}\left(\phi_{\xi}(x)\right)_{j}\right|_{s=0} \cdot\left(\nabla_{j} \psi\right)(x),
\end{aligned}
$$

where $e_{\beta} \in \mathbb{R}^{3 K}$ denotes the unit vector with components $\left(e_{\beta}\right)_{\gamma}=\delta_{\beta, \gamma}$. $U_{\xi}^{-1}$

For $h_{1}, h_{2} \in C_{0}^{\infty}\left(\mathbb{R}^{3 N}\right) \otimes \mathcal{F}$, we calculate the partial derivative using $S_{\xi}=$

$$
\partial_{\beta}\left\langle h_{1}, S_{\xi} h_{2}\right\rangle=\partial_{\beta}\left\langle h_{1},\left|D \phi_{\xi}^{-1}\right|^{1 / 2}\left(h_{2} \circ \phi_{\xi}^{-1}\right)\right\rangle=\left\langle h_{1}, S_{\xi} A_{\beta}(\xi) h_{2}\right\rangle,
$$

where in the second equality we used the product rule of differentiation and the identity $\left|D \phi_{\xi}^{-1}(x)\right|\left|D \phi_{\xi}\left(\phi_{\xi}^{-1}(x)\right)\right|=1$. Integrating (8), we find

$$
\left\langle h_{1}, V_{\xi+t e_{\beta}} h_{2}\right\rangle=\left\langle h_{1}, S_{\xi} h_{2}\right\rangle+\int_{0}^{t}\left\langle h_{1}, V_{\xi+s e_{\beta}} A_{\beta}\left(\xi+s e_{\beta}\right) h_{2}\right\rangle d s .
$$

By an approximation argument using that $|p|$ is a closed operator and that $\mathcal{H} \cap$ $\left(C_{0}^{\infty}\left(\mathbb{R}^{3 N}\right) \otimes \mathcal{F}\right)$ is a core for $|p|$, we conclude that $(9)$ holds for all $h_{2} \in D(|p|)$. For $h_{2} \in D(|p|), \xi \mapsto S_{\xi} A_{\beta}(\xi) h_{2}$ is continuous and therefore (9) holds in fact in the strong sense, i.e.,

$$
V_{\xi+t e_{\beta}} h_{2}=S_{\xi} h_{2}+\int_{0}^{t} V_{\xi+s e_{\beta}} A_{\beta}\left(\xi+s e_{\beta}\right) h_{2} d s, \quad \forall h_{2} \in D(|p|) .
$$

This implies that for all $h_{2} \in D(|p|), t \mapsto V_{\xi+t e_{\beta}} h_{2}$ is $C^{1}$ with derivative $\partial_{\beta} S_{\xi} h_{2}=$ $S_{\xi} A_{\beta}(\xi) h_{2}$.

Step 2: Suppose $\xi \mapsto \psi(\xi)$ is a $C^{1}$ function such that $\psi(\xi) \in D(|p|)$ and $\xi \mapsto$ $\overline{A_{\beta}(\xi)} \psi(\xi)$ is continuous for all $\beta$. Then $\xi \mapsto S_{\xi} \psi(\xi)$ is in $C^{1}$ and for all $\beta$,

$$
\partial_{\beta} S_{\xi} \psi(\xi)=S_{\xi} A_{\beta}(\xi) \psi(\xi)+S_{\xi} \partial_{\beta} \psi(\xi) .
$$

Using the differentiability of $\xi \mapsto \psi(\xi), \psi(\xi) \in D(|p|)$, and Step 1, we see by the product rule of differentiation that $\xi \mapsto S_{\xi} \psi(\xi)$ is differentiable with partial derivative (10). (10) depends continuously on $\xi$, because $\xi \mapsto S_{\xi}$ is strongly continuous and, by assumption, both, $\xi \mapsto \partial_{\beta} \psi_{\xi}$ and $\xi \mapsto A_{\beta}(\xi) \psi_{\xi}$ are continuous.

Step 3: For $\xi$ in a neighborhood of zero:

(a) $\varphi_{\xi} \in D\left(p^{2}\right)$, and the functions $\xi \mapsto p_{\mu} \varphi_{\xi}$ and $\xi \mapsto p_{\mu} p_{\nu} \varphi_{\xi}$ are analytic for all $\mu, \nu$.

(b) For all $\beta, \partial_{\beta} \varphi_{\xi} \in D(|p|)$ and $\xi \mapsto p_{\mu} \partial_{\beta} \varphi_{\xi}$ is analytic for all $\mu$. 
(a) For $h$ from a dense subset of $\mathcal{H},\left\langle h, p_{\nu} p_{\mu} \varphi_{\xi}\right\rangle=\left\langle p_{\nu} p_{\mu} h, \varphi_{\xi}\right\rangle$, which is analytic in $\xi$. Since, by (11) below, $\left\|p_{\mu} p_{\nu} \varphi_{\xi}\right\|$ is locally bounded, the analyticity of $\xi \mapsto p_{\mu} p_{\nu} \varphi_{\xi}$ follows by an approximation argument (Remark III-1.38 in [16]). To prove the bound (11), we use that, for small $\xi, \widetilde{H}(\xi)$ is an analytic family of type (A) and the Coulomb potential is infinitesimally Laplacian bounded, i.e.,

$$
\begin{aligned}
\left\|p^{2} \varphi_{\xi}\right\| & \leq\left\|\left(p^{2}+H_{f}\right) \varphi_{\xi}\right\| \\
& \leq \text { const. }\left(\left\|\widetilde{H}(\xi) \varphi_{\xi}\right\|+\left\|\varphi_{\xi}\right\|\right)=\text { const. }\left(\left\|E(\xi) \varphi_{\xi}\right\|+\left\|\varphi_{\xi}\right\|\right) .
\end{aligned}
$$

The analyticity of $\xi \mapsto p_{\mu} \varphi_{\xi}$ follows using the same arguments as above and the bound $\left\|p_{\mu} \varphi_{\xi}\right\|^{2} \leq\left\|\varphi_{\xi}\right\|\left\|p^{2} \varphi_{\xi}\right\|$.

(b) Since for all $\mu$, the operator $p_{\mu}$ is closed and $\xi \mapsto p_{\mu} \varphi_{\xi}$ is analytic, we have $\partial_{\beta} \varphi_{\xi} \in D(|p|)$ and $\partial_{\beta} p_{\mu} \varphi_{\xi}=p_{\mu} \partial_{\beta} \varphi_{\xi}$.

Step 4: For all $\alpha$, the functions $\xi \mapsto \varphi_{\xi}, \xi \mapsto \partial_{\alpha} \varphi_{\xi}$, and $\xi \mapsto A_{\alpha}(\xi) \varphi_{\xi}$ satisfy the assumptions of Step 2. In particular, $\xi \mapsto S_{\xi} \varphi_{\xi}$ is of class $C^{2}$.

An iteration of Step 2 shows that the statement of the first sentence implies the statement of the second sentence. To prove the former, we recall that analytic functions are of class $C^{\infty}[17]$, and we begin with the following observation. If $\xi \mapsto \psi(\xi) \in D(|p|)$ and $\xi \mapsto p_{\mu} \psi(\xi)$ are in $C^{1}$ for all $\mu$, then $\xi \mapsto A_{\beta}(\xi) \psi(\xi)$ is in $C^{1}$ for all $\beta$, which follows using expression (7). If, moreover, $\psi(\xi) \in D\left(p^{2}\right)$ and $\xi \mapsto$ $p_{\mu} p_{\nu} \psi(\xi)$ is in $C^{1}$ for all $\mu, \nu$, then $A_{\alpha}(\xi) \psi(\xi) \in D(|p|)$ and $\xi \mapsto A_{\beta}(\xi) A_{\alpha}(\xi) \psi(\xi)$ is in $C^{1}$ for all $\alpha, \beta$. Applying, these properties to $\varphi_{\xi}$ and using Step 3 (a), we see that $\xi \mapsto \varphi_{\xi}$ and $\xi \mapsto A_{\alpha}(\xi) \varphi_{\xi}$ satisfy the assumptions of Step 2. Similarly, using Step 3 (b), we see that $\xi \mapsto \partial_{\alpha} \varphi_{\xi}$ satisfies the assumptions of Step 2.

\section{The smooth Feshbach map}

In this section we describe the smooth Feshbach transform of Bach et al. [3] in a slightly generalized form that allows for non self-adjoint smoothed projections. There are further small differences between our presentation here and the one of [3], which are explained in [12].

Let $\chi$ and $\bar{\chi}$ be commuting, nonzero bounded operators, acting on a separable Hilbert space $\mathcal{H}$ and satisfying $\chi^{2}+\bar{\chi}^{2}=1$. By a Feshbach pair $(H, T)$ for $\chi$ we mean a pair of closed operators with same domain

$$
H, T: D(H)=D(T) \subset \mathcal{H} \rightarrow \mathcal{H}
$$

such that $H, T, W:=H-T$, and the operators

$$
\begin{array}{ll}
W_{\chi}:=\chi W \chi, & W_{\bar{\chi}}:=\bar{\chi} W \bar{\chi}, \\
H_{\chi}:=T+W_{\chi}, & H_{\bar{\chi}}:=T+W_{\bar{\chi}},
\end{array}
$$

defined on $D(T)$ satisfy the following assumptions: 
(a) $\chi T \subset T \chi$ and $\bar{\chi} T \subset T \bar{\chi}$,

(b) $T, H_{\bar{\chi}}: D(T) \cap \operatorname{Ran} \bar{\chi} \rightarrow \operatorname{Ran} \bar{\chi}$ are bijections with bounded inverse.

(c) $\bar{\chi} H_{\bar{\chi}}^{-1} \bar{\chi} W \chi: D(T) \subset \mathcal{H} \rightarrow \mathcal{H}$ is a bounded operator.

Henceforth we will call an operator $A: D(A) \subset \mathcal{H} \rightarrow \mathcal{H}$ bounded invertible in a subspace $Y \subset \mathcal{H}$ ( $Y$ not necessarily closed), if $A: D(A) \cap Y \rightarrow Y$ is a bijection with bounded inverse.

\section{Remarks.}

1. To verify (a), it suffices to show that $T \chi=\chi T$ and $T \bar{\chi}=\bar{\chi} T$ on a core of $T$.

2. If $T$ is bounded invertible in $\operatorname{Ran} \bar{\chi},\left\|T^{-1} \bar{\chi} W \bar{\chi}\right\|<1,\left\|\bar{\chi} W T^{-1} \bar{\chi}\right\|<1$ and $T^{-1} \bar{\chi} W \chi$ is bounded, then the bounded invertibility of $H_{\bar{\chi}}$ and condition (c) follow. See Lemma 9 below.

3. Note that $\operatorname{Ran} \chi$ and $\operatorname{Ran} \bar{\chi}$ need not be closed and are not closed in the application to QED. One can however, replace $\operatorname{Ran} \bar{\chi}$ by $\overline{\operatorname{Ran} \bar{\chi}}$ both in condition (b) and in the statement of Theorem 7, below. Then this theorem continues to hold and the proof remains unchanged.

Given a Feshbach pair $(H, T)$ for $\chi$, the operator

$$
F_{\chi}(H, T):=H_{\chi}-\chi W \bar{\chi} H_{\bar{\chi}}^{-1} \bar{\chi} W \chi
$$

on $D(T)$ is called Feshbach map of $H$. The mapping $(H, T) \mapsto F_{\chi}(H, T)$ is called Feshbach map. The auxiliary operators

$$
\begin{aligned}
& Q_{\chi}:=\chi-\bar{\chi} H_{\bar{\chi}}^{-1} \bar{\chi} W \chi \\
& Q_{\chi}^{\#}:=\chi-\chi W_{\bar{\chi}} H_{\bar{\chi}}^{-1} \bar{\chi},
\end{aligned}
$$

play an important role in the analysis of $F_{\chi}(H, T)$. By conditions (a), (c), and the explanation above, they are bounded, and $Q_{\chi}$ leaves $D(T)$ invariant. The Feshbach map is isospectral in the sense of the following Theorem, which generalizes Theorem 2.1 in [3] to non-selfadjoint $\chi$ and $\bar{\chi}$.

Theorem 7. Let $(H, T)$ be a Feshbach pair for $\chi$ on a separable Hilbert space $\mathcal{H}$. Then the following holds:

(i) Let $Y$ be a subspace with $\operatorname{Ran} \chi \subset Y \subset \mathcal{H}$,

$$
T: D(T) \cap Y \rightarrow Y, \quad \text { and } \quad \bar{\chi} T^{-1} \bar{\chi} Y \subset Y .
$$

Then $H: D(H) \subset \mathcal{H} \rightarrow \mathcal{H}$ is bounded invertible if and only if $F_{\chi}(H, T)$ : $D(T) \cap Y \rightarrow Y$ is bounded invertible in $Y$. Moreover,

$$
\begin{aligned}
H^{-1} & =Q_{\chi} F_{\chi}(H, T)^{-1} Q_{\chi}^{\#}+\bar{\chi} H_{\bar{\chi}}^{-1} \bar{\chi}, \\
F_{\chi}(H, T)^{-1} & =\chi H^{-1} \chi+\bar{\chi} T^{-1} \bar{\chi} .
\end{aligned}
$$

(ii) $\chi \operatorname{Ker} H \subset \operatorname{Ker} F_{\chi}(H, T)$ and $Q_{\chi} \operatorname{Ker} F_{\chi}(H, T) \subset \operatorname{Ker} H$. The mappings

$$
\begin{aligned}
\chi & : \operatorname{Ker} H \rightarrow \operatorname{Ker} F_{\chi}(H, T), \\
Q_{\chi} & : \operatorname{Ker} F_{\chi}(H, T) \rightarrow \operatorname{Ker} H,
\end{aligned}
$$

are linear isomorphisms and inverse to each other. 
Remarks.

1. The subspaces $Y=\operatorname{Ran} \chi$ and $Y=\mathcal{H}$ satisfy the conditions stated in (13).

2. From [3] it is known that $\chi$ and $Q_{\chi}$ are one-to-one on $\operatorname{Ker} H$ and $\operatorname{Ker} F_{\chi}(H, T)$ respectively. The stronger result (ii) will be derived from the new algebraic identities (a) and (b) of the following lemma.

Theorem 7 easily follows from the next lemma, which is of interest and importance in its own right.

Lemma 8. Let $(H, T)$ be a Feshbach pair for $\chi$ and let $F:=F_{\chi}(H, T), Q:=Q_{\chi}$, and $Q^{\#}:=Q_{\chi}^{\#}$ for simplicity. Then the following identities hold:

(a) $\left(\bar{\chi} H_{\bar{\chi}}^{-1} \bar{\chi}\right) H=1-Q \chi, \quad$ on $\quad D(T), \quad H\left(\bar{\chi} H_{\bar{\chi}}^{-1} \bar{\chi}\right)=1-\chi Q^{\#}, \quad$ on $\quad \mathcal{H}$,

(b) $\left(\bar{\chi} T^{-1} \bar{\chi}\right) F=1-\chi Q$, on $D(T), \quad F\left(\bar{\chi} T^{-1} \bar{\chi}\right)=1-Q^{\#} \chi$, on $\mathcal{H}$,

(c) $\quad H Q=\chi F, \quad$ on $D(T), \quad Q^{\#} H=F \chi$, on $D(T)$.

For the proofs of Lemma 8 and Theorem 7 we refer to [12].

Lemma 9. Conditions (a), (b), and (c) on Feshbach pairs are satisfied, provided that

(a') $\chi T \subset T \chi$ and $\bar{\chi} T \subset T \bar{\chi}$,

(b') $T$ is bounded invertible in $\operatorname{Ran} \bar{\chi}$,

(c') $\left\|T^{-1} \bar{\chi} W \bar{\chi}\right\|<1,\left\|\bar{\chi} W T^{-1} \bar{\chi}\right\|<1$ and $T^{-1} \bar{\chi} W \chi$ is a bounded operator.

Proof. By assumptions (a') and (b'), on $D(T) \cap \operatorname{Ran} \bar{\chi}$,

$$
H_{\bar{\chi}}=\left(1+\bar{\chi} W T^{-1} \bar{\chi}\right) T,
$$

and $T: D(T) \cap \operatorname{Ran} \bar{\chi} \rightarrow \operatorname{Ran} \bar{\chi}$ is a bijection with bounded inverse. From (c') it follows that

$$
1+\bar{\chi} W T^{-1} \bar{\chi}: \operatorname{Ran} \bar{\chi} \rightarrow \operatorname{Ran} \bar{\chi}
$$

is a bijection with bounded inverse. In fact, $\left(1+\bar{\chi} W T^{-1} \bar{\chi}\right) \operatorname{Ran} \bar{\chi} \subset \operatorname{Ran} \bar{\chi}$, the Neumann series

$$
\sum_{n \geq 0}\left(-\bar{\chi} W T^{-1} \bar{\chi}\right)^{n}=1-\bar{\chi} W T^{-1} \bar{\chi} \sum_{n \geq 0}\left(-\bar{\chi} W T^{-1} \bar{\chi}\right)^{n}
$$

converges, and it maps Ran $\bar{\chi}$ to Ran $\bar{\chi}$. Hence $H_{\bar{\chi}}\lceil\operatorname{Ran} \bar{\chi}$ is bounded invertible.

Finally, from $H_{\bar{\chi}}=T\left(1+T^{-1} W_{\bar{\chi}}\right)$ and (c') it follows that

$$
H_{\bar{\chi}}^{-1} \bar{\chi} W \chi=\left(1+T^{-1} W_{\bar{\chi}}\right)^{-1} T^{-1} \bar{\chi} W \chi,
$$

which, again by (c'), is bounded. 


\section{The initial Hamiltonian on Fock space}

As explained in the introduction, the first step in our renormalization analysis of $H_{g}(s)$ is to use the Feshbach map to define an isospectral operator $H^{(0)}[s, z]$ on

$$
\mathcal{H}_{\text {red }}:=P_{[0,1]}\left(H_{f}\right) \mathcal{F} \text {. }
$$

Let $\chi, \bar{\chi} \in C^{\infty}(\mathbb{R} ;[0,1])$ with $\chi(t)=1$ if $t \leq 3 / 4, \chi(t)=0$ if $t \geq 1$ and $\chi^{2}+\bar{\chi}^{2}=1$. For $\rho>0$ we define operators $\chi_{\rho}:=\chi\left(H_{f} / \rho\right), \bar{\chi}_{\rho}:=\bar{\chi}\left(H_{f} / \rho\right)$, and

$$
\begin{aligned}
& \chi(s):=P_{\mathrm{at}}(s) \otimes \chi_{1}, \\
& \bar{\chi}(s):=\bar{P}_{\mathrm{at}}(s) \otimes 1+P_{\mathrm{at}}(s) \otimes \bar{\chi}_{1} .
\end{aligned}
$$

By assumption on $\chi$ and $\bar{\chi}$,

$$
\chi(s)^{2}+\bar{\chi}(s)^{2}=1,
$$

but $\chi(s)$ and $\overline{\boldsymbol{\chi}}(s)$ will not be self-adjoint unless $s$ is real. Since $P_{\text {at }}(s)$ is a bounded projection with one-dimensional range, any linear operator $L$ in $\mathcal{H}_{\mathrm{at}} \otimes \mathcal{F}$ that is defined and bounded on $\operatorname{Ran} P_{\text {at }}(s) \otimes \mathcal{F}$, defines a unique bounded linear transformation $\langle L\rangle_{\text {at }, s}$ on $\mathcal{F}$, through the equation

$$
\left(P_{\text {at }}(s) \otimes 1\right) L\left(P_{\text {at }}(s) \otimes 1\right)=P_{\text {at }}(s) \otimes\langle L\rangle_{\text {at }, s} .
$$

We are no ready to define the effective Hamiltonian $H^{(0)}[s, z]$ on $\mathcal{H}_{\text {red }}$. To this end we assume, for the moment, that $\left(H_{g}(s)-z, H_{0}(s)-z\right)$ is a Feshbach pair for $\chi(s)$. This assumption will be justified by Theorem 13 below. From $1=$ $P_{\text {at }}(s)+\bar{P}_{\text {at }}(s)$ and the fact that $P_{\text {at }}(s)$ is a rank one operator, we find

$$
F_{\boldsymbol{\chi}(s)}\left(H_{g}(s)-z, H_{0}(s)-z\right)=\left(H_{0}(s)-z\right) \bar{P}_{\mathrm{at}}(s) \otimes 1+P_{\mathrm{at}}(s) \otimes \tilde{H}^{(0)}[s, z],
$$

with

$$
\tilde{H}^{(0)}[s, z]=E_{\text {at }}(s)-z+H_{f}+W_{\text {at }}[s, z]
$$

and $W_{\text {at }}[s, z] \in \mathcal{L}(\mathcal{F})$ given by

$$
\begin{aligned}
W_{\mathrm{at}}[s, z]= & g\left\langle\chi_{1} W(s) \chi_{1}\right\rangle_{\mathrm{at}, s} \\
& -g^{2}\left\langle\chi_{1} W(s) \overline{\boldsymbol{\chi}}(s)\left(H_{g}(s)-z\right)_{\overline{\boldsymbol{\chi}}(s)}^{-1} \overline{\boldsymbol{\chi}}(s) W(s) \chi_{1}\right\rangle_{\mathrm{at}, s} .
\end{aligned}
$$

The operators $\tilde{H}^{(0)}[s, z]$ and $H_{g}(s)-z$ are isospectral in the sense of Theorem 7 . More explicitly, the following proposition holds true.

Proposition 10. Let $\left(H_{g}(s)-z, H_{0}(s)-z\right)$ be a Feshbach pair for $\boldsymbol{\chi}(s)$. Then:

(i) $H_{g}(s)-z: D\left(H_{0}(s)\right) \subset \mathcal{H} \rightarrow \mathcal{H}$ is bounded invertible if and only if $\tilde{H}^{(0)}[s, z]$ is bounded invertible on $\mathcal{H}_{\text {red }}$.

(ii) The following maps are linear isomorphisms and inverses of each other:

$$
\begin{aligned}
\chi(s): \operatorname{Ker}\left(H_{g}(s)-z\right) & \longrightarrow P_{\mathrm{at}}(s) \mathcal{H}_{\mathrm{at}} \otimes \operatorname{Ker} \tilde{H}^{(0)}[s, z], \\
Q_{\boldsymbol{\chi}(s)}: P_{\mathrm{at}}(s) \mathcal{H}_{\mathrm{at}} \otimes \operatorname{Ker} \tilde{H}^{(0)}[s, z] & \longrightarrow \operatorname{Ker}\left(H_{g}(s)-z\right) .
\end{aligned}
$$


Proof. (i) We fix $(s, z)$ and for notational simplicity suppress the $s$ and $z$ dependence. Let $Y=\operatorname{Ran}\left(P_{\text {at }} \otimes P_{[0,1]}\left(H_{f}\right)\right)$. Then $H_{0}: D\left(H_{0}\right) \cap Y \rightarrow Y$ and $\bar{\chi}\left(H_{0}-z\right)^{-1} \bar{\chi} Y \subset Y$. By Theorem 7 ,

$$
\begin{aligned}
& H_{g}-z \text { is bounded invertible in } \mathcal{H} \\
& \quad \Leftrightarrow \quad F_{\chi}\left(H_{g}-z, H_{0}-z\right) \text { is bounded invertible in } Y \\
& \quad \Leftrightarrow \quad \tilde{H}^{(0)} \text { is bounded invertible on } \mathcal{H}_{\text {red }},
\end{aligned}
$$

where the last equivalence follows from $F_{\chi}\left(H_{g}-z, H_{0}-z\right)=1 \otimes \tilde{H}^{(0)}$ on $Y$.

Statement (ii) follows immediately from Theorem 7, (ii).

Since $\operatorname{Ran} \chi(s) \subset P_{\text {at }}(s) \mathcal{H}_{\text {at }} \otimes \mathcal{H}_{\text {red }}$, Proposition 10 (ii) implies that

$$
\operatorname{Ker} \tilde{H}^{(0)}[s, z]=\operatorname{Ker} \tilde{H}^{(0)}[s, z] \cap \mathcal{H}_{\text {red }}=\operatorname{Ker}\left(\tilde{H}^{(0)}[s, z]\left\lceil\mathcal{H}_{\text {red }}\right) .\right.
$$

Therefore, and because of Proposition 10 (i), it is sufficient for our purpose to study the restriction

$$
H^{(0)}[s, z]:=\tilde{H}^{(0)}[s, z] \uparrow \mathcal{H}_{\text {red }} .
$$

In the remainder of this section we use Hypotheses I-III to verify, for small $g$, the assumption of Proposition 10 and to show that $H^{(0)}[s, z]$ is analytic on $\mathcal{U}$. To this end we need the following lemmas.

Lemma 11. Suppose that Hypotheses I-III hold. Then, for all $(s, z) \in \mathcal{U}, H_{0}(s)-z$ is bounded invertible on $\operatorname{Ran} \bar{\chi}(s)$ and

$$
\begin{aligned}
\sup _{(s, z) \in \mathcal{U}}\left\|\left(H_{f}+1\right)\left(H_{0}(s)-z\right)^{-1} \overline{\boldsymbol{\chi}}(s)\right\| & <\infty \\
\sup _{(s, z) \in \mathcal{U}}\left\|W(s)\left(H_{0}(s)-z\right)^{-1} \overline{\boldsymbol{\chi}}(s)\right\| & <\infty \\
& \sup _{(s, z) \in \mathcal{U}}\left\|\left(H_{0}(s)-z\right)^{-1} \overline{\boldsymbol{\chi}}(s) W(s)\right\|<\infty .
\end{aligned}
$$

Proof. The parameter $s$ is suppressed in this proof to make long expressions more readable. Recall that $\overline{\boldsymbol{\chi}} H_{0} \subset H_{0} \overline{\boldsymbol{\chi}}$. Hence $H_{0}-z$ maps $D\left(H_{0}\right) \cap \operatorname{Ran} \overline{\boldsymbol{\chi}}$ into $\operatorname{Ran} \overline{\boldsymbol{\chi}}$. Moreover,

$$
\operatorname{Ran} \bar{\chi}=\operatorname{Ran}\left(\bar{P}_{\mathrm{at}} \otimes 1\right) \oplus \operatorname{Ran}\left(P_{\mathrm{at}} \otimes \bar{\chi}_{1}\right)
$$

where

$$
\begin{aligned}
& H_{0}-z: \quad D\left(H_{0}\right) \cap \operatorname{Ran}\left(\bar{P}_{\mathrm{at}} \otimes 1\right) \rightarrow \operatorname{Ran}\left(\bar{P}_{\mathrm{at}} \otimes 1\right), \\
& H_{0}-z \quad: \quad D\left(H_{0}\right) \cap \operatorname{Ran}\left(P_{\mathrm{at}} \otimes \bar{\chi}_{1}\right) \rightarrow \operatorname{Ran}\left(P_{\mathrm{at}} \otimes \bar{\chi}_{1}\right) .
\end{aligned}
$$

Working in a spectral representation where $H_{f}$ is multiplication by $q \geq 0$, it is easily seen from Hypothesis III that (21) and (22) are bounded invertible for $(s, z) \in \mathcal{U}$, and hence that $\left(H_{0}-z\right): D\left(H_{0}\right) \cap \operatorname{Ran} \bar{\chi} \rightarrow \operatorname{Ran} \bar{\chi}$ is a bijection. The 
inverses of (21) and (22) are bounded by

$$
\begin{array}{r}
\left\|\left(H_{0}-z\right)^{-1} \bar{P}_{\text {at }} \otimes 1\right\| \leq \sup _{(s, z) \in \mathcal{U}} \sup _{q \geq 0}\left\|\left(H_{\text {at }}-z+q\right)^{-1} \bar{P}_{\text {at }}\right\|, \\
\left\|\left(H_{0}-z\right)^{-1} P_{\text {at }} \otimes \chi\left(H_{f} \geq 3 / 4\right)\right\| \leq \sup _{(s, z) \in \mathcal{U}} \sup _{q \geq 3 / 4}\left|\frac{1}{E_{\text {at }}-z+q}\right|\left\|P_{\text {at }}\right\| .
\end{array}
$$

Since $\bar{\chi}=\left[\bar{P}_{\text {at }} \otimes 1\right] \bar{\chi}+\left[P_{\text {at }} \otimes \chi\left(H_{f} \geq 3 / 4\right)\right] \bar{\chi}$ it follows from (23), (24) and Hypothesis III that

$$
\sup _{(s, z) \in \mathcal{U}}\left\|\left(H_{0}-z\right)^{-1} \uparrow \operatorname{Ran} \bar{\chi}\right\|<\infty .
$$

Bound (20) is proved in a similar way, using

$$
\begin{array}{r}
\left\|\left(H_{f}+1\right)\left(H_{0}-z\right)^{-1} \bar{P}_{\text {at }} \otimes 1\right\| \leq \sup _{(s, z) \in \mathcal{U}} \sup _{q \geq 0}\left\|\frac{q+1}{H_{\mathrm{at}}-z+q} \bar{P}_{\mathrm{at}}\right\|, \\
\left\|\left(H_{f}+1\right)\left(H_{0}-z\right)^{-1} P_{\text {at }} \otimes \chi\left(H_{f} \geq 3 / 4\right)\right\| \leq \sup _{(s, z) \in \mathcal{U}} \sup _{q \geq 3 / 4}\left|\frac{q+1}{E_{\mathrm{at}}-z+q}\right|\left\|P_{\mathrm{at}}\right\|,
\end{array}
$$

instead of (23) and (24). The right sides of (25) and (26) are finite by Hypothesis III.

The remaining inequalities of Lemma 11 follow from (20) and

$$
\begin{aligned}
& \sup _{s}\left\|W(s)\left(H_{f}+1\right)^{-1}\right\| \leq \sup _{s}\left\|G_{s}\right\|_{\omega}, \\
& \sup _{s}\left\|\left(H_{f}+1\right)^{-1} W(s)\right\| \leq \sup _{s}\left\|G_{s}\right\|_{\omega},
\end{aligned}
$$

where $\sup _{s}\left\|G_{s}\right\|_{\omega}<\infty$ by Hypothesis I.

Lemma 12. The mapping $s \mapsto W(s)\left(H_{f}+1\right)^{-1 / 2} \in \mathcal{L}(\mathcal{H})$ is analytic on $V$.

Proof. From

$$
\left\|W(s)\left(H_{f}+1\right)^{-1 / 2}\right\| \leq 2\left\|G_{s}\right\|_{\omega}
$$

we see, by Hypothesis I, that $s \mapsto W(s)\left(H_{f}+1\right)^{-1 / 2}$ is uniformly bounded. By this uniform bound (see Theorem III-3.12 of [16]) it is sufficient to show that the function

$$
s \mapsto\left(\psi_{1}, W(s)\left(H_{f}+1\right)^{-1 / 2} \psi_{2}\right),
$$

is analytic on $V$, for all $\psi_{1}, \psi_{2}$ in the dense linear subspace spanned by all vectors of the form $\varphi \otimes S_{n}\left(h_{1} \otimes h_{2} \otimes \cdots \otimes h_{n}\right)$, with $\varphi \in \mathcal{H}_{\text {at }}$ and $h_{i} \in \mathfrak{h}, n \in \mathbb{N}$. For such vectors, $(27)$ is a linear combination of terms of the form $\left(\varphi_{1} \otimes h, G_{s} \varphi_{2}\right)$, with $\varphi_{1}, \varphi_{2} \in \mathcal{H}_{\text {at }}$ and $h \in \mathfrak{h}$. They are analytic by Hypothesis I.

Theorem 13. Suppose Hypotheses I-III hold, and let $\mathcal{U} \subset V \times \mathbb{C}$ be given by Hypothesis III. Then there exists a $g_{0}>0$ such that for all $(s, z) \in \mathcal{U}$ and for all $g \in\left[0, g_{0}\right)$, the pair $\left(H_{g}(s)-z, H_{0}(s)-z\right)$ is a Feshbach pair for $\chi(s)$. Moreover, $H_{g}^{(0)}[s, z]$ is analytic on $\mathcal{U}$. 
Proof. To prove that $H_{g}(s)=H_{0}(s)+g W(s)$ is closed on $D\left(H_{0}(s)\right)$ for all $g \in \mathbb{R}$, we prove that $W(s)$ is infinitesimally bounded with respect to $H_{0}(s)$. Suppose that $(s, z) \in \mathcal{U}$ for some $z \in \mathbb{C}$. By Hypothesis I,

$$
\left\|W(s)\left(H_{f}+1\right)^{-1 / 2}\right\| \leq 2\left\|G_{s}\right\|_{\omega}<\infty .
$$

On the other hand, by the reasoning in the proof of (20), Hypothesis III implies that $z-q \in \rho\left(H_{\text {at }}(s)\right)$ for $q \geq 1$, that $w:=z-1 \in \rho\left(H_{0}(s)\right)$, and that

$$
H_{f}\left(H_{0}(s)-w\right)^{-1} \in \mathcal{L}(\mathcal{H}) .
$$

Combining (28) and (29) we see that, for all $\varphi \in D\left(H_{\mathrm{at}}(s)\right) \otimes D\left(H_{f}\right)$,

$$
\begin{aligned}
\|W(s) \varphi\|^{2} & \leq C_{0}\left\langle\varphi,\left(H_{f}+1\right) \varphi\right\rangle \\
& =C_{0}\left\langle\varphi,\left(H_{f}+1\right)\left(H_{0}(s)-w\right)^{-1}\left(H_{0}(s)-w\right) \varphi\right\rangle \\
& \leq C_{1}\|\varphi\|\left\|H_{0}(s) \varphi\right\|+C_{2}\|\varphi\|^{2} \\
& \leq C_{1} \varepsilon\left\|H_{0}(s) \varphi\right\|^{2}+\left(\frac{C_{1}}{\varepsilon}+C_{2}\right)\|\varphi\|^{2}
\end{aligned}
$$

with constants $C_{0}, C_{1}, C_{2}$.

Next we verify the criteria for Feshbach pairs from Lemma 9. Obviously, $\chi(s) H_{0}(s)=H_{0}(s) \chi(s)$ and $\overline{\boldsymbol{\chi}}(s) H_{0}(s)=H_{0}(s) \overline{\boldsymbol{\chi}}(s)$ on $D\left(H_{\mathrm{at}}\right) \otimes D\left(H_{f}\right)$. By the first remark of Section 4 , this proves condition (a') of Lemma 9.

By Lemma 11, $H_{0}(s)-z$ is bounded invertible on $\operatorname{Ran} \overline{\boldsymbol{\chi}}(s)$ and

$$
\begin{aligned}
& \sup _{(s, z) \in U}\left\|g \bar{\chi}(s) W(s)\left(H_{0}(s)-z\right)^{-1} \bar{\chi}(s)\right\|<1, \\
& \sup _{(s, z) \in U}\left\|\left(H_{0}(s)-z\right)^{-1} \bar{\chi}(s) g W(s) \bar{\chi}(s)\right\|<1,
\end{aligned}
$$

for $g$ sufficiently small. This proves (b') and (c') of Lemma 9 and hence completes the proof that $\left(H_{g}(s)-z, H_{0}(s)-z\right)$ is a Feshbach pair.

It remains to prove the analyticity of $H^{(0)}[s, z]\left\lceil\mathcal{H}_{\text {red }}\right.$. By $(17), H^{(0)}(s, z)$ is analytic if $W_{\text {at }}[s, z]$ is analytic. We will show that

$$
(s, z) \mapsto \chi_{1}\left(g W(s)-g^{2} W(s) \bar{\chi}(s)\left(H_{g}(s)-z\right)_{\bar{\chi}(s)}^{-1} \bar{\chi}(s) W(s)\right) \chi_{1}
$$

is analytic in $s$ and $z$. By Eqns. (16) and (19) this will imply the analyticity of $\left\langle\alpha, W_{\text {at }}[s, z] \beta\right\rangle$ for all $\alpha, \beta \in \mathcal{F}$, which, by Theorem 3.12 of Chapter III in [16], proves that $W_{\text {at }}[s, z]$ is analytic in $s$ and $z$.

Since $\chi_{1} W(s)$ and $W(s) \chi_{1}$ are analytic the analyticity of (31) follows if we show that

$$
(s, z) \mapsto\left(H_{g}(s)-z\right)_{\bar{\chi}(s)}^{-1} \bar{\chi}(s)
$$

is analytic. Assuming that $|g|$ is small enough for (30) to hold, the Neumann series

$$
\left.\left(H_{g}-z\right)_{\bar{\chi}}^{-1}\right|_{\operatorname{Ran} \bar{\chi}}=\left.\left(H_{0}-z\right)^{-1} \sum_{n=0}^{\infty}\left(-\bar{\chi} g W\left(H_{0}-z\right)^{-1} \bar{\chi}\right)^{n}\right|_{\operatorname{Ran} \bar{\chi}}
$$


converges uniformly for $(s, z) \in \mathcal{U}$. Hence $(32)$ will be analytic if each term of the series (33) is analytic. By Lemma $12, W(s)\left(H_{f}+1\right)^{-1}$ is analytic. Hence it remains to prove analyticity of

$$
\left.\left(H_{f}+1\right)\left(H_{0}(s)-z\right)^{-1} \bar{\chi}(s)\right|_{\operatorname{Ran} \bar{\chi}(s)} .
$$

By the definition of $\bar{\chi}(s)$,

$$
\begin{aligned}
& \left(H_{f}+1\right)\left(H_{0}(s)-z\right)^{-1} \bar{\chi}(s) \\
= & \left(H_{f}+1\right)\left(H_{0}(s)-z\right)^{-1}\left(\bar{P}_{\mathrm{at}}(s) \otimes 1\right)+\left(H_{f}+1\right)\left(E_{\mathrm{at}}(s)+H_{f}-z\right)^{-1}\left(P_{\mathrm{at}}(s) \otimes \bar{\chi}_{1}\right) .
\end{aligned}
$$

The factor $\left(H_{f}+1\right)\left(E_{\mathrm{at}}(s)+H_{f}-z\right)^{-1}$ in the second term on the r.h.s. can be viewed as a composition of analytic functions. The analyticity of the first term on the r.h.s. is derived, in a spectral representation of $H_{f}$, from Hypothesis III, and Proposition 27 of the appendix.

\section{The renormalization transformation}

The renormalization transformation is defined on a subset of $\mathcal{L}\left(\mathcal{H}_{\text {red }}\right)$ that is parameterized by vectors of a Banach space $\mathcal{W}_{\xi}=\oplus_{m, n \geq 0} \mathcal{W}_{m, n}$. We begin with the definition of this Banach space.

The Banach space $\mathcal{W}_{0,0}$ is the space of continuously differentiable functions

$$
\begin{aligned}
\mathcal{W}_{0,0} & :=C^{1}([0,1]) \\
\|w\| & :=\|w\|_{\infty}+\left\|w^{\prime}\right\|_{\infty}
\end{aligned}
$$

where $w^{\prime}(r):=\partial_{r} w(r)$. For $m, n \in \mathbb{N}$ with $m+n \geq 1$ and $\mu>0$ we set

$$
\begin{aligned}
\mathcal{W}_{m, n} & :=L_{s}^{2}\left(B^{m+n}, \frac{d K}{|K|^{2+2 \mu}} ; \mathcal{W}_{0,0}\right) \\
\left\|w_{m, n}\right\|_{\mu} & :=\left(\int_{B^{m+n}}\left\|w_{m, n}(K)\right\|^{2} \frac{d K}{|K|^{2+2 \mu}}\right)^{1 / 2}
\end{aligned}
$$

where $B:=\left\{k \in \mathbb{R}^{3} \times\{1,2\}:|k| \leq 1\right\}$ and

$$
|K|:=\prod_{j=1}^{m+n}\left|k_{j}\right|, \quad d K:=\prod_{j=1}^{m+n} d k_{j} .
$$

That is, $\mathcal{W}_{m, n}$ is the space of measurable functions $w_{m, n}: B^{m+n} \rightarrow \mathcal{W}_{0,0}$ that are symmetric with respect to all permutations of the $m$ arguments from $B^{m}$ and the $n$ arguments from $B^{n}$, respectively, such that $\left\|w_{m, n}\right\|_{\mu}$ is finite.

For given $\xi \in(0,1)$ and $\mu>0$ we define a Banach space

$$
\begin{aligned}
\mathcal{W}_{\xi} & :=\bigoplus_{m, n \in \mathbb{N}} \mathcal{W}_{m, n} \\
\|w\|_{\mu, \xi} & :=\sum_{m, n \geq 0} \xi^{-(m+n)}\left\|w_{m, n}\right\|_{\mu},
\end{aligned}
$$


$\left\|w_{0,0}\right\|_{\mu}:=\left\|w_{0,0}\right\|$, as the completion of the linear space of finite sequences $w=$ $\left(w_{m, n}\right)_{m, n \in \mathbb{N}} \in \bigoplus_{m, n \in \mathbb{N}} \mathcal{W}_{m, n}$ with respect to the norm $\|w\|_{\mu, \xi}$. The spaces $\mathcal{W}_{m, n}$ will often be identified with the corresponding subspaces of $\mathcal{W}_{\xi}$.

Next we define a linear mapping $H: \mathcal{W}_{\xi} \rightarrow \mathcal{L}\left(\mathcal{H}_{\text {red }}\right)$. For finite sequences $w=\left(w_{m, n}\right) \in \mathcal{W}_{\xi}$ the operator $H(w)$ is the sum

$$
H(w):=\sum_{m, n} H_{m, n}(w)
$$

of operators $H_{m, n}(w)$ on $\mathcal{H}_{\text {red }}$, defined by $H_{0,0}(w):=w_{0,0}\left(H_{f}\right)$, and, for $m+n \geq 1$,

$$
H_{m, n}(w):=P_{\text {red }}\left(\int_{B^{m+n}} a^{*}\left(k^{(m)}\right) w_{m, n}\left(H_{f}, K\right) a\left(\tilde{k}^{(n)}\right) d K\right) P_{\text {red }},
$$

where $P_{\text {red }}:=P_{[0,1]}\left(H_{f}\right), K=\left(k^{(m)}, \tilde{k}^{(n)}\right)$, and

$$
\begin{aligned}
k^{(m)} & =\left(k_{1}, \ldots, k_{m}\right) \in\left(\mathbb{R}^{3} \times\{1,2\}\right)^{m}, & & a^{*}\left(k^{(m)}\right)=\prod_{i=1}^{m} a^{*}\left(k_{i}\right), \\
\tilde{k}^{(n)} & =\left(\tilde{k}_{1}, \ldots, \tilde{k}_{n}\right) \in(\mathbb{R} \times\{1,2\})^{n}, & a\left(\tilde{k}^{(n)}\right) & =\prod_{i=1}^{n} a\left(\tilde{k}_{i}\right) .
\end{aligned}
$$

By the continuity established in the following proposition, the mapping $w \mapsto H(w)$ has a unique extension to a bounded linear transformation on $\mathcal{W}_{\xi}$.

Proposition 14 ([3]).

(i) For all $\mu>0, m, n \in \mathbb{N}$, with $m+n \geq 1$, and $w \in \mathcal{W}_{m, n}$,

$$
\left\|H_{m, n}(w)\right\| \leq\left\|\left(P_{\Omega}^{\perp} H_{f}\right)^{-m / 2} H_{m, n}(w)\left(P_{\Omega}^{\perp} H_{f}\right)^{-n / 2}\right\| \leq \frac{1}{\sqrt{m^{m} n^{n}}}\left\|w_{m, n}\right\|_{\mu} .
$$

(ii) For all $\mu>0$ and all $w \in \mathcal{W}_{\xi}$

$$
\begin{aligned}
\|H(w)\| & \leq\|w\|_{\mu, \xi} \\
\|H(w)\| & \leq \xi\|w\|_{\mu, \xi}, \quad \text { if } \quad w_{0,0}=0 .
\end{aligned}
$$

In particular, the mapping $w \mapsto H(w)$ is continuous.

Proof. Statement (ii) follows immediately from (i) and $\xi \leq 1$. For (i) we refer to [3], Theorem 3.1.

Given $\alpha, \beta, \gamma \in \mathbb{R}_{+}$we define neighborhoods, $\mathcal{B}(\alpha, \beta, \gamma) \subset H\left(\mathcal{W}_{\xi}\right)$ of the operator $P_{\text {red }} H_{f} P_{\text {red }} \in \mathcal{L}\left(\mathcal{H}_{\text {red }}\right)$ by

$$
\mathcal{B}(\alpha, \beta, \gamma):=\left\{H(w)|| w_{0,0}(0) \mid \leq \alpha,\left\|w_{0,0}^{\prime}-1\right\|_{\infty} \leq \beta,\left\|w-w_{0,0}\right\|_{\mu, \xi} \leq \gamma\right\} .
$$

Note that $w_{0,0}(0)=\left\langle\Omega, w_{0,0}\left(H_{f}\right) \Omega\right\rangle=\langle\Omega, H(w) \Omega\rangle$. The definition of $\mathcal{B}(\alpha, \beta, \gamma)$ is motivated by the following Lemma and by Theorem 16 .

Lemma 15. Suppose $\rho, \xi \in(0,1)$ and $\mu>0$. If $H(w) \in \mathcal{B}(\rho / 2, \rho / 8, \rho / 8)$, then $\left(H(w), H_{0,0}(w)\right)$ is a Feshbach pair for $\chi_{\rho}$. 
Proof. The assumption $H(w) \in \mathcal{B}(\rho / 2, \rho / 8, \rho / 8)$ implies, by Proposition 14 , that

$$
\left\|H(w)-H_{0,0}(w)\right\| \leq \xi \frac{\rho}{8} .
$$

For $r \in\left[\frac{3}{4} \rho, 1\right]$,

$$
\begin{aligned}
\left|w_{0,0}(r)\right| & \geq r-\left|\left(w_{0,0}(r)-w_{0,0}(0)\right)-r\right|-\left|w_{0,0}(0)\right| \\
& \geq r\left(1-\sup _{r}\left|w_{0,0}^{\prime}(r)-1\right|\right)-\frac{\rho}{2} \\
& \geq \frac{3 \rho}{4}\left(1-\frac{\rho}{8}\right)-\frac{\rho}{2} \geq \frac{\rho}{8} .
\end{aligned}
$$

By the spectral theorem,

$$
\left\|H_{0,0}(w)^{-1} \nmid \operatorname{Ran} \bar{\chi}_{\rho}\right\|=\| w_{0,0}\left(H_{f}\right)^{-1}\left\lceil\operatorname{Ran} \bar{\chi}_{\rho} \| \leq \sup _{r \in\left[\frac{3}{4} \rho, 1\right]} \frac{1}{\left|w_{0,0}(r)\right|} \leq \frac{8}{\rho} .\right.
$$

Since $\left\|\bar{\chi}_{\rho}\right\| \leq 1$, it follows from the estimates above that

$$
\| H_{0,0}(w)^{-1} \bar{\chi}_{\rho}\left(H(w)-H_{0,0}(w)\right) \bar{\chi}_{\rho}\left\lceil\operatorname{Ran} \bar{\chi}_{\rho} \| \leq \xi<1 .\right.
$$

This implies the bounded invertibility of

$$
\begin{aligned}
&\left(H_{0,0}(w)+\bar{\chi}_{\rho}\left(H(w)-H_{0,0}(w)\right) \bar{\chi}_{\rho}\right)\left\lceil\operatorname{Ran} \bar{\chi}_{\rho}\right. \\
&=H_{0,0}(w)\left(1+H_{0,0}(w)^{-1} \bar{\chi}_{\rho}\left(H(w)-H_{0,0}(w)\right) \bar{\chi}_{\rho}\right) \uparrow \operatorname{Ran} \bar{\chi}_{\rho} .
\end{aligned}
$$

The other conditions on a Feshbach pair are now also satisfied, since $H(w)-$ $H_{0,0}(w)$ is bounded on $\mathcal{H}_{\text {red }}$.

The renormalization transformation we use is a composition of a Feshbach transformation and a unitary scaling that puts the operator back on the original Hilbert space $\mathcal{H}_{\text {red }}$. Unlike the renormalization transformation of Bach et al [3], our renormalization transformation involves no analytic transformation of the spectral parameter.

Given $\rho \in(0,1)$, let $\mathcal{H}_{\rho}=\operatorname{Ran} \chi\left(H_{f} \leq \rho\right)$. Let $w \in \mathcal{W}_{\xi}$ and suppose $\left(H(w), H_{0,0}(w)\right)$ is a Feshbach pair for $\chi_{\rho}$. Then

$$
F_{\chi_{\rho}}\left(H(w), H_{0,0}(w)\right): \mathcal{H}_{\rho} \rightarrow \mathcal{H}_{\rho}
$$

is iso-spectral with $H(w)$ in the sense of Theorem 7. In order to get a isospectral operator on $\mathcal{H}_{\text {red }}$, rather than $\mathcal{H}_{\rho}$, we use the linear isomorphism

$$
\Gamma_{\rho}: \mathcal{H}_{\rho} \rightarrow \mathcal{H}_{1}=\mathcal{H}_{\mathrm{red}}, \quad \Gamma_{\rho}:=\Gamma\left(U_{\rho}\right) \uparrow \mathcal{H}_{\rho},
$$

where $U_{\rho} \in \mathcal{L}\left(L^{2}\left(\mathbb{R}^{3} \times\{1,2\}\right)\right)$ is defined by

$$
\left(U_{\rho} f\right)(k):=\rho^{3 / 2} f(\rho k) .
$$

Note that $\Gamma_{\rho} H_{f} \Gamma_{\rho}^{*}=\rho H_{f}$, and hence $\Gamma_{\rho} \chi_{\rho} \Gamma_{\rho}^{*}=\chi_{1}$. The renormalization transformation $\mathcal{R}_{\rho}$ maps bounded operators on $\mathcal{H}_{\text {red }}$ to bounded linear operators on $\mathcal{H}_{\text {red }}$ 
and is defined on those operators $H(w)$ for which $\left(H(w), H_{0,0}(w)\right)$ is a Feshbach pair with respect to $\chi_{\rho}$. Explicitly,

$$
\mathcal{R}_{\rho}(H(w)):=\rho^{-1} \Gamma_{\rho} \mathcal{F}_{\chi_{\rho}}\left(H(w), H_{0,0}(w)\right) \Gamma_{\rho}^{*},
$$

which is a bounded linear operator on $\mathcal{H}_{\text {red }}$. In [3], Theorem 3.3, it is shown that $w \mapsto H(w)$ is one-to-one. Hence $w \in \mathcal{W}_{\xi}$ is uniquely determined by the operator $H(w)$ and the domain of $\mathcal{R}_{\rho}$, as described above, is a well-defined subset of $\mathcal{L}\left(\mathcal{H}_{\text {red }}\right)$. By Lemma 15 it contains the ball $\mathcal{B}(\rho / 2, \rho / 8, \rho / 8)$.

The following theorem describes conditions under which the Renormalization transform may be iterated.

Theorem 16 (BCFS [3]). There exists a constant $C_{\chi} \geq 1$ depending only on $\chi$, such that the following holds. If $\mu>0, \rho \in(0,1), \xi=\sqrt{\rho} /\left(4 C_{\chi}\right)$, and $\beta, \gamma \leq \rho /\left(8 C_{\chi}\right)$, then

$$
\mathcal{R}_{\rho}-\rho^{-1}\langle\cdot\rangle_{\Omega}: \mathcal{B}(\rho / 2, \beta, \gamma) \rightarrow \mathcal{B}\left(\alpha^{\prime}, \beta^{\prime}, \gamma^{\prime}\right)
$$

where

$$
\alpha^{\prime}=C_{\beta} \frac{\gamma^{2}}{\rho}, \quad \beta^{\prime}=\beta+C_{\beta} \frac{\gamma^{2}}{\rho}, \quad \gamma^{\prime}=C_{\gamma} \rho^{\mu} \gamma,
$$

with $C_{\beta}:=\frac{3}{2} C_{\chi}, C_{\gamma}:=128 C_{\chi}^{2}$.

This theorem is a variant of Theorem 3.8 of [3], with additional information from the proof of that theorem, in particular from Equations (3.104), (3.107) and (3.109). Another difference is due to our different definition of the renormalization transformation, i.e., without analytic deformation of the spectral parameter.

\section{Renormalization preserves analyticity}

This section provides one of the key tools for our method to work, Proposition 17 below, which implies that analyticity is preserved under renormalization. It is part (a) of the following proposition that is nontrivial and not proved in the papers of Bach et al. (see Theorem 2.5 of [3] and the remark thereafter).

Proposition 17. Let $S$ be an open subset of $\mathbb{C}^{\nu+1}, \nu \geq 0$. Suppose $\sigma \mapsto H\left(w^{\sigma}\right) \in$ $\mathcal{L}\left(\mathcal{H}_{\text {red }}\right)$ is analytic on $S$, and that $H\left(w^{\sigma}\right)$ belongs to some ball $\mathcal{B}(\alpha, \beta, \gamma)$ for all $\sigma \in S$. Then:

(a) $H_{0,0}\left(w^{\sigma}\right)$ is analytic on $S$.

(b) If for all $\sigma \in S,\left(H\left(w^{\sigma}\right), H_{0,0}\left(w^{\sigma}\right)\right)$ is a Feshbach pair for $\chi_{\rho}$, then $F_{\chi_{\rho}}\left(H\left(w^{\sigma}\right), H_{0,0}\left(w^{\sigma}\right)\right)$ is analytic on $S$.

Proof. Suppose (a) holds true. Then $H_{0,0}\left(w^{\sigma}\right)$ and $W=H\left(w^{\sigma}\right)-H_{0,0}\left(w^{\sigma}\right)$ are analytic function of $\sigma \in S$ and hence so is the Feshbach map

$$
\begin{aligned}
F_{\chi_{\rho}}\left(H\left(w^{\sigma}\right)\right. & \left., H_{0,0}\left(w^{\sigma}\right)\right) \\
& =H_{0,0}\left(w^{\sigma}\right)+\chi_{\rho} W \chi_{\rho}-\chi_{\rho} W \bar{\chi}_{\rho}\left(H_{0,0}\left(w^{\sigma}\right)+\bar{\chi}_{\rho} W \bar{\chi}_{\rho}\right)^{-1} \bar{\chi}_{\rho} W \chi_{\rho} .
\end{aligned}
$$

This proves (b) and it remains to prove (a). 
Recall from Section 6 that $B=\left\{k \in \mathbb{R}^{3} \times\{1,2\}:|k| \leq 1\right\}$ and let $P_{1}$ denote the projection onto the one boson subspace of $\mathcal{H}_{\text {red }}$, which is isomorphic to $L^{2}(B)$. Then $P_{1} H\left(w^{\sigma}\right) P_{1}$, like $H\left(w^{\sigma}\right)$, is analytic and

$$
\begin{aligned}
P_{1} H\left(w^{\sigma}\right) P_{1} & =P_{1} H_{0,0}\left(w^{\sigma}\right) P_{1}+P_{1} H_{1,1}\left(w^{\sigma}\right) P_{1} \\
& =D_{\sigma}+K_{\sigma},
\end{aligned}
$$

where $D_{\sigma}$ denotes multiplication with $w_{0,0}^{\sigma}$ and $K_{\sigma}$ is the Hilbert Schmidt operator with kernel

$$
M_{\sigma}(k, \tilde{k})=w_{1,1}^{\sigma}(0, k, \tilde{k}) .
$$

Our strategy is to show first that $K_{\sigma}$ and hence $P_{1} H_{0,0}\left(w^{\sigma}\right) P_{1}=P_{1} H\left(w^{\sigma}\right) P_{1}-K_{\sigma}$ is analytic. Then we show that $H_{0,0}\left(w^{\sigma}\right)$ is an analytic operator on $\mathcal{H}_{\text {red }}$.

Step 1: $K_{\sigma}$ is analytic. that

For each $n \in \mathbb{N}$ let $\left\{Q_{i}^{(n)}\right\}_{i}$ be a collection of $n$ measurable subsets of $B$ such

$$
B=\bigcup_{i=1}^{n} Q_{i}^{(n)}, \quad Q_{i}^{(n)} \cap Q_{j}^{(n)}=\emptyset, \quad i \neq j,
$$

and

$$
\left|Q_{i}^{(n)}\right| \leq \frac{\text { const }}{n} .
$$

Let $\chi_{i}^{(n)}$ denote the operator on $L^{2}(B)$ of multiplication with $\chi_{Q_{i}^{(n)}}$. Then for $i \neq j$, $\chi_{i}^{(n)} D_{\sigma} \chi_{j}^{(n)}=0$ because $\chi_{i}^{(n)}$ and $\chi_{j}^{(n)}$ have disjoint support and commute with $D_{\sigma}$. Together with (35) this implies that

$$
\chi_{i}^{(n)} K_{\sigma} \chi_{j}^{(n)}=\chi_{i}^{(n)} P_{1} H\left(w^{\sigma}\right) P_{1} \chi_{j}^{(n)}, \quad \text { for } \quad i \neq j .
$$

Since the right hand side is analytic, so is the left hand side and hence

$$
K_{\sigma}^{(n)}=\sum_{i \neq j} \chi_{i}^{(n)} K_{\sigma} \chi_{j}^{(n)}
$$

is analytic. It follows that $\sigma \mapsto\left\langle\varphi, K_{\sigma}^{(n)} \psi\right\rangle$ is analytic for all $\varphi, \psi$ in $L^{2}(B)$. Now let $\varphi, \psi \in C(B)$. Then

$$
\begin{aligned}
& \left|\left\langle\varphi, K_{\sigma}^{(n)} \psi\right\rangle-\left\langle\varphi, K_{\sigma} \psi\right\rangle\right| \\
& \quad=\left|\int_{B \times B} \bar{\varphi}(x) \psi(y) M_{\sigma}(x, y) \sum_{i=1}^{n} \chi_{i}^{(n)}(x) \chi_{i}^{(n)}(y) d x d y\right| \\
& \quad \leq\|\varphi\|_{\infty}\|\psi\|_{\infty}\left\|K_{\sigma}\right\|_{\mathrm{HS}}\left(\sum_{i=1}^{n}\left|Q_{i}^{(n)}\right|^{2}\right)^{1 / 2} \longrightarrow 0, \quad(n \rightarrow \infty),
\end{aligned}
$$

uniformly in $\sigma$, because the Hilbert Schmidt norm $\left\|K_{\sigma}\right\|_{\mathrm{HS}}$ is bounded uniformly in $\sigma$ (in fact, it is bounded by $\gamma$ ). This proves that $\left\langle\varphi, K_{\sigma} \psi\right\rangle$ is analytic for all $\varphi, \psi \in C(B)$. Since $C(B)$ is dense in $L^{2}(B)$, an other approximate argument using 
$\sup _{\sigma}\left\|K_{\sigma}\right\|<\infty$ shows that $\left\langle\varphi, K_{\sigma} \psi\right\rangle$ is analytic for all $\varphi, \psi \in L^{2}(B)$. Therefore $\sigma \mapsto K_{\sigma}$ is analytic [16].

Step 2: For each $k \in B, w_{0,0}^{\sigma}(|k|)$ is an analytic function of $\sigma$.

For each $n \in \mathbb{N}$ let $f_{k, n} \in L^{2}(B)$ denote a multiple of the characteristic function of $B_{1 / n}(k) \cap B$ with $\left\|f_{n, k}\right\|=1$. By the continuity of $w_{0,0}^{\sigma}(|k|)$ as a function of $k$

$$
\begin{aligned}
w_{0,0}^{\sigma}(|k|) & =\lim _{n \rightarrow \infty} \int_{B}\left|f_{k, n}(x)\right|^{2} w_{0,0}^{\sigma}(|x|) d x \\
& =\lim _{n \rightarrow \infty}\left\langle a^{*}\left(f_{k, n}\right) \Omega, H_{0,0}\left(w^{\sigma}\right) a^{*}\left(f_{k, n}\right) \Omega\right\rangle .
\end{aligned}
$$

Since $a^{*}\left(f_{k, n}\right) \Omega \in P_{1} \mathcal{H}_{\text {red }}$ the expression $\langle\cdots\rangle$, before taking the limit, is an analytic function of $\sigma$. By assumption on $w_{0,0}^{\sigma}$, this function is Lipschitz continuous with respect to $|k|$ uniformly in $\sigma$. Therefore the convergence in (38) is uniform in $\sigma$ and hence $w_{0,0}^{\sigma}(|k|)$ is analytic by the Weierstrass approximation theorem from complex analysis.

Step 3: $H_{0,0}\left(w^{\sigma}\right)=w_{0,0}^{\sigma}\left(H_{f}\right)$ is analytic.

By the spectral theorem

$$
\left\langle\varphi, w_{0,0}^{\sigma}\left(H_{f} P_{\mathrm{red}}\right) \varphi\right\rangle=\int_{[0,1]} w_{0,0}^{\sigma}(\lambda) d \mu_{\varphi}(\lambda) .
$$

By an application of Lebesgue's dominated convergence theorem, using $\sup _{\sigma}\left\|w_{0,0}^{\sigma}\right\|$ $<\infty$, we see that the right hand side, we call it $\varphi(\sigma)$, it is a continuous function of $\sigma$. Therefore

$$
\int_{\Gamma} \varphi(\sigma) d \sigma=\int_{[0,1]}\left(\int_{\Gamma} w_{0,0}^{\sigma}(\lambda) d \sigma\right) d \mu_{\varphi}(\lambda)
$$

for all closed loops $\Gamma: t \mapsto \sigma(t)$ in $S$, with $\sigma_{j}$ constant for all but one $j \in$ $\{1, \ldots, \nu+1\}$. The analyticity of $\sigma \mapsto \varphi(\sigma)$ now follows from the analyticity of $w_{0,0}^{\sigma}(\lambda)$ and the theorems of Cauchy and Morera. By polarization, $w_{0,0}^{\sigma}\left(H_{f} P_{\text {red }}\right)$ is weakly analytic and hence analytic.

\section{Iterating the renormalization transform}

In Section 5 we have reduced, for small $|g|$, the problem of finding an eigenvalue of $H_{g}(s)$ in the neighborhood $U_{0}(s):=\{z \in \mathbb{C} \mid(s, z) \in \mathcal{U}\}$ of $E_{\text {at }}(s)$ to finding $z \in \mathbb{C}$ such that $H^{(0)}[s, z]$ has a non-trivial kernel. We now use the renormalization map to define a sequence $H^{(n)}[s, z]:=\mathcal{R}^{n} H^{(0)}[s, z]$ of operators on $\mathcal{H}_{\text {red }}$, which, by Theorem 7, are isospectral in the sense that $\operatorname{Ker} H^{(n+1)}[s, z]$ is isomorphic to $\operatorname{Ker} H^{(n)}[s, z]$. The main purpose of the present section is to show that the operators $H^{(n)}[s, z]$ are well-defined for all $z$ from non-empty, but shrinking sets $U_{n}(s) \searrow$ $\left\{z_{\infty}(s)\right\},(n \rightarrow \infty)$. In the next section it will turn out that $H^{(n)}\left[s, z_{\infty}(s)\right]$ has a non-trivial kernel and hence that $z_{\infty}(s)$ is an eigenvalue of $H_{g}(s)$. The construction 
of the sets $U_{n}(s)$ is based on Theorems 13 and 23, but not on the explicit form of $H^{(0)}[s, z]$ as given by (18). Moreover, this construction is pointwise in $s$ and $g$, all estimates being uniform in $s \in V$ and $|g|<g_{0}$ for some $g_{0}>0$. We therefore drop these parameters from our notations and we now explain the construction of $H^{(n)}[z]$ making only the following assumption:

(A) $U_{0}$ is an open subset of $\mathbb{C}$ and for every $z \in U_{0}$,

$$
H^{(0)}[z] \in \mathcal{B}(\infty, \rho / 8, \rho / 8) \text {. }
$$

The polydisc $\mathcal{B}(\infty, \rho / 8, \rho / 8) \subset H\left(\mathcal{W}_{\xi}\right)$ is defined in terms of $\xi:=\sqrt{\rho} /\left(4 C_{\chi}\right)$ and $\mu>0$, where $\rho \in(0,1)$ and $C_{\chi}$ is given by Theorem 16 .

By Lemma 15 , we may define $H^{(1)}[z], \ldots, H^{(N)}[z]$, recursively by

$$
H^{(n)}[z]:=\mathcal{R}_{\rho}\left(H^{(n-1)}[z]\right)
$$

provided that $H^{(0)}[z], \ldots, H^{(N-1)}[z]$ belong to $\mathcal{B}(\rho / 2, \rho / 8, \rho / 8)$. Theorem 16 gives us sufficient conditions for this to occur: by iterating the map $(\beta, \gamma) \mapsto\left(\beta^{\prime}, \gamma^{\prime}\right)$ starting with $\left(\beta_{0}, \gamma_{0}\right)$, we find the conditions

$$
\begin{array}{r}
\gamma_{n}:=\left(C_{\gamma} \rho^{\mu}\right)^{n} \gamma_{0} \leq \rho /\left(8 C_{\chi}\right) \\
\beta_{n}:=\beta_{0}+\left(\frac{C_{\beta}}{\rho} \sum_{k=0}^{n-1}\left(C_{\gamma} \rho^{\mu}\right)^{2 k}\right) \gamma_{0}^{2} \leq \rho /\left(8 C_{\chi}\right),
\end{array}
$$

for $n=0, \ldots, N-1$. They are obviously satisfied for all $n \in \mathbb{N}$ if $C_{\gamma} \rho^{\mu}<1$ and if $\beta_{0}, \gamma_{0}$ are sufficiently small. Let this be the case and let

$$
E^{(n)}(z):=\left\langle\Omega, H^{(n)}[z] \Omega\right\rangle .
$$

Then it remains to make sure that $\left|E^{(n)}(z)\right| \leq \rho / 2$ for $n=0, \ldots, N-1$. This is achieved by adjusting the admissible values of $z$ step by step. We define recursively, for all $n \geq 1$,

$$
U_{n}:=\left\{z \in U_{n-1}:\left|E^{(n-1)}(z)\right| \leq \rho / 2\right\} .
$$

If $z \in U_{N}, H^{(0)}(z) \in \mathcal{B}\left(\infty, \beta_{0}, \gamma_{0}\right)$, and $\rho, \beta_{0}, \gamma_{0}$ are small enough, as explained above, then the operators $H^{(n)}(z)$ for $n=1, \ldots, N$ are well defined by (39). In addition we know from Theorem 16 that $H^{(n)}(z) \in \mathcal{B}\left(\infty, \beta_{n}, \gamma_{n}\right)$, and that

$$
\left|E^{(n)}(z)-\frac{E^{(n-1)}(z)}{\rho}\right| \leq \frac{C_{\beta}}{\rho} \gamma_{n-1}^{2}=: \alpha_{n} .
$$

This latter information will be used in the proof of Lemma 19 to show that the sets $U_{n}$ are not empty. We summarize:

Lemma 18. Suppose that (A) holds with $\rho \in(0,1)$ so small, that $C_{\gamma} \rho^{\mu}<1$. Suppose $\beta_{0}, \gamma_{0} \leq \rho /\left(8 C_{\chi}\right)$ and, in addition,

$$
\beta_{0}+\frac{C_{\beta} / \rho}{1-\left(C_{\gamma} \rho^{\mu}\right)^{2}} \gamma_{0}^{2} \leq \frac{\rho}{8 C_{\chi}} .
$$


If $H^{(0)}[z] \in \mathcal{B}\left(\infty, \beta_{0}, \gamma_{0}\right)$ for all $z \in U_{0}$, then $H^{(n)}[z]$ is well defined for $z \in U_{n}$, and

$$
H^{(n)}[z]-\frac{1}{\rho} E^{(n-1)}(z) \in \mathcal{B}\left(\alpha_{n}, \beta_{n}, \gamma_{n}\right), \quad \text { for } \quad n \geq 1
$$

with $\alpha_{n}, \beta_{n}$, and $\gamma_{n}$ as in (40), (41), and (43).

The next lemma establishes conditions under which the set $U_{0}$ and $U_{n}$ are non-empty. We introduce the discs

and note that $U_{n}=E^{(n-1)^{-1}}\left(D_{\rho / 2}\right)$.

$$
D_{r}:=\{z \in \mathbb{C}|| z \mid \leq r\}
$$

Lemma 19. Suppose that (A) holds with $U_{0} \ni E_{\text {at }}$ and $\rho \in(0,4 / 5)$ so small that $C_{\gamma} \rho^{\mu}<1$ and $\overline{B\left(E_{\mathrm{at}}, \rho\right)} \subset U_{0}$. Suppose that $\alpha_{0}<\rho / 2, \beta_{0}, \gamma_{0} \leq \rho /\left(8 C_{\chi}\right)$ and that (44) hold. If $z \mapsto H^{(0)}[z] \in \mathcal{L}\left(\mathcal{H}_{\mathrm{at}}\right)$ is analytic in $U_{0}$ and $H^{(0)}[z]-\left(E_{\mathrm{at}}-z\right) \in$ $\mathcal{B}\left(\alpha_{0}, \beta_{0}, \gamma_{0}\right)$ for all $z \in U_{0}$, then the following is true.

(a) For $n \geq 0, E^{(n)}: U_{n} \rightarrow \mathbb{C}$ is analytic in $U_{n}^{\circ}$ and a conformal map from $U_{n+1}$ onto $D_{\rho / 2}$. In particular, $E^{(n)}$ has a unique zero, $z_{n}$, in $U_{n}$. Moreover,

$$
B\left(E_{\text {at }}, \rho\right) \supset U_{1} \supset U_{2} \supset U_{3} \supset \cdots \text {. }
$$

(b) The limit $z_{\infty}:=\lim _{n \rightarrow \infty} z_{n}$ exists and for $\epsilon:=1 / 2-\rho / 2-\alpha_{1}>0$,

$$
\left|z_{n}-z_{\infty}\right| \leq \rho^{n} \exp \left(\frac{1}{2 \rho \epsilon^{2}} \sum_{k=0}^{\infty} \alpha_{k}\right) .
$$

Remark. We call a function $f: A \rightarrow B$ conformal if it is the restriction of an analytic bijection $f: U \rightarrow V$ between open sets $U \supset A$ and $V \supset B$, and $f(A)=B$.

Proof. Since $H^{(0)}$ is analytic on $U_{0}$, it follows, by Theorem 17 , that $H^{(n)}$ is analytic on $U_{n}^{\circ}$ for all $n \in \mathbb{N}$. In particular $E^{(n)}$ is analytic on $U_{n}^{\circ}$. To begin with we prove:

$\left(\boldsymbol{I}_{1}^{\prime}\right) \quad U_{1} \subset B\left(E_{\mathrm{at}}, \rho\right)$ and $E^{(0)}: U_{1} \rightarrow D_{\rho / 2}$ conformally .

By assumption on $H^{(0)}(z)$,

$$
\left|E^{(0)}(z)-\left(E_{\text {at }}-z\right)\right| \leq \alpha_{0}, \quad \forall z \in U_{0} .
$$

Hence, if $z \in E^{(0)^{-1}}\left(D_{\rho / 2+\epsilon}^{\circ}\right)$ then

$$
\left|E_{\text {at }}-z\right| \leq \alpha_{0}+\rho / 2+\epsilon<\rho,
$$

provided $\epsilon>0$ is chosen sufficiently small. This proves that $U_{1} \subset E^{(0)^{-1}}\left(D_{\rho / 2+\epsilon}^{\circ}\right) \subset$ $B\left(E_{\text {at }}, \rho\right)$. Since $E^{(0)}$ is continuous, it follows that $E^{(0)^{-1}}\left(D_{\rho / 2+\epsilon}^{\circ}\right)$ is open in $\mathbb{C}$. If

$$
E^{(0)}: E^{(0)^{-1}}\left(D_{\rho / 2+\epsilon}^{\circ}\right) \rightarrow D_{\rho / 2+\epsilon}^{\circ} \quad \text { is a bijection, }
$$

then it is conformal on $U_{1}$. So it suffices to prove (47). To this end we use Rouche's theorem. Let $w \in D_{\rho / 2+\epsilon}^{\circ}$. Then $E_{\text {at }}-z-w$ has exactly one zero $z \in B\left(E_{\text {at }}, \rho\right)$ and for all $z \in \partial B\left(E_{\text {at }}, \rho\right)$,

$$
\left|E_{\text {at }}-z-w\right| \geq \rho-|w| \geq \rho / 2>\alpha_{0} .
$$


Since, by (46),

$$
\left|\left(E^{(0)}(z)-w\right)-\left(E_{\text {at }}-z-w\right)\right| \leq \alpha_{0},
$$

for all $z \in \overline{B\left(E_{\text {at }}, \rho\right)}$, it follows that $E^{(0)}(z)-w$, like $\left(E_{\text {at }}-z-w\right)$ has exactly one zero $z \in \overline{B\left(E_{\text {at }}, \rho\right)}$. This proves $(47)$ because $E^{(0)^{-1}}\left(D_{\rho / 2+\epsilon}^{\circ}\right) \subset B\left(E_{\text {at }}, \rho\right)$.

Next we prove, by induction in $n$, that

$\left(\boldsymbol{I}_{\boldsymbol{n}}\right) \quad E^{(n-1)}: U_{n} \rightarrow D_{\rho / 2}$ conformally

For $n=1$, this follows from $I_{1}^{\prime}$. Suppose $I_{n}$, holds. First note that $\alpha_{n} \leq \alpha_{1}=$ $\left(C_{\beta} / \rho\right) \gamma_{0}^{2}$, Ineq. (44), $C_{\chi} \geq 1$, and $\rho<4 / 5$ imply

$$
\alpha_{n}+\rho / 2<1 / 2 .
$$

Thus we can choose a positive $\epsilon$ such that

$$
\alpha_{n}+\rho / 2+2 \epsilon<1 / 2 .
$$

We define $D_{+}^{\circ}:=D_{\rho / 2+\epsilon}^{\circ}$ and $D_{-}^{\circ}:=D_{\rho / 2-\rho \epsilon}^{\circ}$, so that $D_{-}^{\circ} \subset D_{\rho / 2} \subset D_{+}^{\circ}$. We claim that

$$
E^{(n)^{-1}}\left(D_{+}^{\circ}\right) \subset E^{(n-1)^{-1}}\left(D_{-}^{\circ}\right)
$$

and that

$$
E^{(n)}: E^{(n)^{-1}}\left(D_{+}^{\circ}\right) \rightarrow\left(D_{+}^{\circ}\right) \quad \text { is a bijection . }
$$

Suppose (50) and (51) hold. Then by (50) and the induction Hypothesis $I_{n}$, $E^{(n)^{-1}}\left(D_{+}^{\circ}\right) \subset U_{n}^{\circ}$. Since $E^{(n)}$ is continuous on $U_{n}^{\circ}$, it follows that $E^{(n)^{-1}}\left(D_{+}^{\circ}\right)$ is open. Since $E^{(n)}$ is analytic, (51) implies $I_{n+1}$. It remains to prove (50) and (51).

(50) follows from (45) and (49): if $\left|E^{(n)}(z)\right|<\rho / 2+\epsilon$ and $\mid E^{(n)}(z)-\rho^{-1}$ $E^{(n-1)}(z) \mid \leq \alpha_{n}$, then $\left|E^{(n-1)}(z)\right|<\rho / 2-\rho \epsilon$.

To prove (51) we use Rouche's Theorem. Let $w \in D_{+}^{\circ}$. Then, by (49), $\rho w \in$ $D_{-}^{\circ}$ and the induction Hypothesis $I_{n}$ implies that $E^{(n-1)}(z)-\rho w$ has exactly one zero $z \in E^{(n-1)^{-1}}\left(D_{-}^{\circ}\right)$. On the other hand, by (49),

$$
\left|\rho^{-1}\left(E^{(n-1)}(z)-\rho w\right)\right| \geq \rho^{-1}\left|E^{(n-1)}(z)\right|>\alpha_{n}, \quad \forall z \in \partial\left(E^{(n-1)^{-1}}\left(D_{-}^{\circ}\right)\right) .
$$

Since, by (45),

$$
\left|\left(E^{(n)}(z)-w\right)-\rho^{-1}\left(E^{(n-1)}(z)-\rho w\right)\right| \leq \alpha_{n}, \quad \forall z \in U_{n},
$$

it follows that $E^{(n)}(z)-w$, like $E^{(n-1)}(z)-\rho w$, has exactly one zero $z \in E^{(n-1)^{-1}}$ $\left(D_{-}^{\circ}\right)$. Therefore, (51) follows from (50).

(b) By (a), $U_{k+1}$ contains $z_{k}$ and all subsequent terms of the sequence $\left(z_{n}\right)_{n=1}^{\infty}$. Thus, to prove that $\left(z_{n}\right)_{n=1}^{\infty}$ converges, it suffices to show that the diameter of $U_{n}$ tends to zero as $n$ tends to infinity. To this end, let $F^{(k)}$ denote the inverse of the 
function $E^{(k)}: U_{k+1} \rightarrow D_{\rho / 2}$. Then

$$
\begin{aligned}
\operatorname{diam}\left(U_{n+1}\right) & =\operatorname{diam}\left(F^{(n)}\left(D_{\rho / 2}\right)\right) \\
& =\operatorname{diam}\left(E^{(\text {at })} \circ F^{(0)} \circ E^{(0)} \cdots \circ F^{(n-1)} \circ E^{(n-1)} \circ F^{(n)}\left(D_{\rho / 2}\right)\right),
\end{aligned}
$$

where we used that $z \mapsto E^{(\text {at) }}(z):=E_{\text {at }}-z$ is an isometry. We want to estimate (52) from above. Let $k \geq 1$. For all $z \in D_{\rho / 2}$, by (45),

$$
\left|\rho z-E^{(k-1)}\left(F^{(k)}(z)\right)\right| \leq \rho \alpha_{k},
$$

and hence $\left|E^{(k-1)} \circ F^{(k)}(z)\right| \leq \rho \alpha_{k}+\rho^{2} / 2 \leq \rho / 2-\epsilon \rho$, where $\epsilon:=1 / 2-\rho / 2-\alpha_{1}$ is positive by (48). This shows that $E^{(k-1)} \circ F^{(k)}$ maps $D_{\rho / 2}$ into $D_{\rho / 2-\rho \epsilon}$. By Cauchy's integral formula and by (53),

$$
\begin{aligned}
\left|\partial_{z}\left(E^{(k-1)} \circ F^{(k)}(z)-\rho z\right)\right| & =\left|\frac{1}{2 \pi i} \int_{\partial D_{\rho / 2}} \frac{E^{(k-1)} \circ F^{(k)}(w)-\rho w}{(z-w)^{2}} d w\right| \\
& \leq \alpha_{k} /\left(2 \epsilon^{2}\right), \text { for } z \in D_{\rho / 2-\rho \epsilon} .
\end{aligned}
$$

It follows that $\left|\left(E^{(k-1)} \circ F^{(k)}\right)^{\prime}(z)\right|=\rho+\alpha_{k} /\left(2 \epsilon^{2}\right)$ for $z \in D_{\rho / 2-\rho \epsilon}$. A similar estimate yields $\left|\left(E^{(\text {at })} \circ F^{(0)}\right)^{\prime}(z)\right| \leq 1+\alpha_{0} /\left(2 \rho \epsilon^{2}\right)$ for $z \in D_{\rho / 2-\rho \epsilon}$. Using these estimates and (52) we obtain

$$
\begin{aligned}
\operatorname{diam}\left(U_{n+1}\right) & \leq\left(1+\alpha_{0} /\left(2 \rho \epsilon^{2}\right)\right) \operatorname{diam}\left(E^{(0)} \circ F^{(1)} \circ \cdots \circ F^{(n-1)}\left(D_{\rho / 2-\rho \epsilon}\right)\right) \\
& \leq \rho^{n-1} \prod_{k=0}^{n-1}\left(1+\alpha_{k} /\left(2 \rho \epsilon^{2}\right)\right) \operatorname{diam} D_{\rho / 2-\rho \epsilon} \\
& \leq \rho^{n} \exp \left(\sum_{k=0}^{\infty} \alpha_{k} /\left(2 \rho \epsilon^{2}\right)\right),
\end{aligned}
$$

where we used that $1+x \leq \exp (x)$ in the last inequality. This proves (b).

The following results will allow us to show that $z_{\infty}(s)=\inf \sigma(H(s))$, if $s \in \mathbb{R}$.

Corollary 20. Suppose the assumptions of Lemma 19 hold, $E_{\mathrm{at}} \in \mathbb{R}$, and $H^{(0)}(z)^{*}=$ $H^{(0)}(\bar{z})$ for all $z \in \overline{B\left(E_{\text {at }}, \rho\right)}$. Then for all $n \geq 0, U_{n+1} \cap \mathbb{R}$ is an interval and $\partial_{x} E^{(n)}(x)<0$ on $U_{n+1} \cap \mathbb{R}$.

Proof. Using an induction argument and the definition of the renormalization transformation one sees that $H^{(n)}(z)^{*}=H^{(n)}(\bar{z})$ for $z \in U_{n}$. In particular,

$$
\overline{E^{(n)}(z)}=E^{(n)}(\bar{z}) \text { for all } z \in U_{n} \text {. }
$$

This together with $E^{(n)}: U_{n+1} \rightarrow D_{\rho / 2}$ being a homeomorphism, c.f. Lemma 19, implies that

$$
\left[a_{n+1}, b_{n+1}\right]:=\left(E^{(n)}\right)^{-1}[-\rho / 2, \rho / 2]=U_{n+1} \cap \mathbb{R}
$$

is indeed an interval. Moreover, by Lemma 19 ,

$$
E_{\text {at }}-\rho<a_{1}<a_{2}<\cdots \leq z_{\infty} .
$$


We prove by induction that for all $n \in \mathbb{N}$,

$$
\partial_{x} E^{(n)}(x)<0 \quad \text { on }\left[a_{n+1}, b_{n+1}\right] .
$$

We begin with $n=0$. By assumption on $H^{(0)}[z],\left|E^{(0)}(z)-\left(E_{\text {at }}-z\right)\right| \leq \alpha_{0}$ for $z \in U_{0}$. For $z=E_{\text {at }}-\rho$, which belongs to $U_{0}$ by choice of $\rho$, we obtain

$$
\left|E^{(0)}\left(E_{\text {at }}-\rho\right)-\rho\right| \leq \alpha_{0}<\frac{1}{2} \rho,
$$

by assumption on $\alpha_{0}$. This proves that $E^{(0)}\left(E_{\text {at }}-\rho\right)>\rho / 2$. Since $\left|E^{(0)}(x)\right| \geq \rho / 2$ for $x \in\left[E_{\text {at }}-\rho, a_{1}\right]$ the function $E^{(0)}$ must be positive on this interval. On the other hand it is a diffeomorphism from $\left[a_{1}, b_{1}\right]$ onto $[-\rho / 2, \rho / 2]$ by Lemma 19 . It follows that $\partial_{x} E^{(0)}(x)<0$ for $x \in\left[a_{1}, b_{1}\right]$.

To prove (55) for $n \geq 1$ suppose that

$$
\partial_{x} E^{(n-1)}(x)<0 \quad \text { on }\left[a_{n}, b_{n}\right] .
$$

Let $F^{(n)}$ be the inverse of $E^{(n)}: U_{n+1} \rightarrow D_{\rho / 2}$. Setting $z=0$ in (54) we obtain

$$
\left|\partial_{x}\left(E^{(n-1)} \circ F^{(n)}(x)-\rho x\right)\right|_{x=0} \mid \leq \frac{\rho}{2} \frac{\rho \alpha_{n}}{(\rho / 2)^{2}} \leq 2 \alpha_{1}<\rho .
$$

This shows that

$$
\begin{aligned}
0 & <\left(E^{(n-1)} \circ F^{(n)}\right)^{\prime}(0) \\
& =\left(\partial_{x} E^{(n-1)}\right)\left(F^{(n)}(0)\right) \frac{1}{\left(\partial_{x} E^{(n)}\right)\left(F^{(n)}(0)\right)} .
\end{aligned}
$$

Hence $\left(\partial_{x} E^{(n)}\right)\left(F^{(n)}(0)\right)$ has the same sign as $\left(\partial_{x} E^{(n-1)}\right)\left(F^{(n)}(0)\right)$, which is negative by induction hypothesis $(56)$. Since $E^{(n)}:\left[a_{n+1}, b_{n+1}\right] \rightarrow[-\rho / 2, \rho / 2]$ is a diffeomorphism, $\partial_{x} E^{(n)}(x)<0$ for all $x \in\left[a_{n+1}, b_{n+1}\right]$.

Proposition 21. Suppose the assumptions of Lemma 19 are satisfied, $E_{\text {at }}$ is real and $H^{(0)}[z]^{*}=H^{(0)}[\bar{z}]$ for $z \in \overline{B\left(E_{\mathrm{at}}, \rho\right)}$. Then, there exists an $a<z_{\infty}$ such that $H^{(0)}[x]$ has a bounded inverse for $x \in\left(a, z_{\infty}\right)$.

Proof. Let $\left[a_{n}, b_{n}\right]=U_{n} \cap \mathbb{R}$, c.f. Corollary 20. Then, by Lemma 19, $a_{1}<a_{2}<$ $a_{3}<\cdots<z_{\infty}$ and $\lim _{n \rightarrow \infty} a_{n}=z_{\infty}$. We show that $H^{(n)}[x]$ is bounded invertible for $x \in\left[a_{n}, a_{n+1}\right)$. By a repeated application of the Feshbach property, Theorem 7 (i), it will follow that $H^{(n-1)}[x], \ldots, H^{(0)}[x]$ are also bounded invertible for $x \in\left[a_{n}, a_{n+1}\right)$.

Let $x \in\left[a_{n}, a_{n+1}\right)$. Then both $H^{(n)}[x]$ and $H_{0,0}^{(n)}[x]$ are self-adjoint and, by (34) and (45),

$$
H^{(n)}[x]=H_{0,0}^{(n)}[x]+\left(H^{(n)}[x]-H_{0,0}^{(n)}[x]\right) \geq E^{(n)}(x)-\xi \gamma_{n},
$$

where we have used that $H_{0,0}^{(n)}[x] \geq E^{(n)}(x)$, which follows from $\beta_{n}<1$. Since the function $E^{(n)}$ is decreasing on $\left[a_{n+1}, b_{n+1}\right]$ with a zero in this interval, we know that $E^{(n)}\left(a_{n+1}\right)>0$. On the other hand, by construction of $U_{n},\left|E^{(n)}\right| \geq \rho / 2$ on $\left[a_{n}, a_{n+1}\right)$. Therefore $(57)$ implies that $H^{(n)}[x] \geq\left(\rho / 2-\xi \gamma_{n}\right)>(\rho / 2-\xi \rho / 8)>0$, which proves that $H^{(n)}[x]$ is bounded invertible. 


\section{Construction of the eigenvector}

Next we show that zero is an eigenvalue of $H^{(0)}\left[z_{\infty}\right]$. In fact, we will show that zero is an eigenvalue of $H^{(n)}\left[z_{\infty}\right]$ for every $n \in \mathbb{N}$. To this end we define

$$
Q_{n}[z]:=\chi_{\rho}-\bar{\chi}_{\rho}\left(H_{\bar{\chi}_{\rho}}^{(n)}[z]\right)^{-1} \bar{\chi}_{\rho} W^{(n)}[z] \chi_{\rho}, \quad \text { for } \quad z \in U_{n}
$$

where $W^{(n)}=H^{(n)}-H_{0,0}^{(n)}$. By the definition of $H^{(n)}[z]$ and by Lemma 8 (c),

$$
H^{(n-1)}[z] Q_{n-1}[z] \Gamma_{\rho}^{*}=\left(\rho \Gamma_{\rho}^{*} \chi_{1}\right) H^{(n)}[z]
$$

and moreover, if $H^{(n)}[z] \varphi=0$ and $\varphi \neq 0$ then $Q_{n-1}[z] \Gamma_{\rho}^{*} \varphi \neq 0$ by Theorem 7 . Thus if 0 is an eigenvalue of $H^{(n)}[z]$, then it is an eigenvalue of $H^{(n-1)}[z]$ as well, and the operator $Q_{n-1}[z] \Gamma_{\rho}^{*}$ maps the corresponding eigenvectors of $H^{(n)}[z]$ to eigenvectors of $H^{(n-1)}[z]$.

Theorem 22. Suppose the assumptions of Lemma 19 hold. Then the limit

$$
\varphi^{(0)}=\lim _{n \rightarrow \infty} Q_{0}\left[z_{\infty}\right] \Gamma_{\rho}^{*} Q_{1}\left[z_{\infty}\right] \ldots \Gamma_{\rho}^{*} Q_{n}\left[z_{\infty}\right] \Omega
$$

exists, $\varphi^{(0)} \neq 0$ and $H^{(0)}\left[z_{\infty}\right] \varphi^{(0)}=0$. Moreover,

$$
\left\|\varphi^{(0)}-Q_{0}\left[z_{\infty}\right] \Gamma_{\rho}^{*} Q_{1}\left[z_{\infty}\right] \ldots \Gamma_{\rho}^{*} Q_{n}\left[z_{\infty}\right] \Omega\right\| \leq C \sum_{l=n+1}^{\infty} \gamma_{l},
$$

where $C=C\left(\rho, \xi, \gamma_{0}\right)$.

Remark. By Theorem 22 and by Proposition 10 (ii), $Q_{\chi}\left(\varphi_{\text {at }} \otimes \varphi^{(0)}\right)$ is an eigenvector of $H_{g}$ with eigenvalue $z_{\infty}$.

Proof. For $k, l \in \mathbb{N}$ with $k \leq l$ we define $\varphi_{k, l} \in \mathcal{H}_{\text {red }}$ by

$$
\varphi_{k, l}:=\left(Q_{k}\left[z_{\infty}\right] \Gamma_{\rho}^{*}\right)\left(Q_{k+1}\left[z_{\infty}\right] \Gamma_{\rho}^{*}\right) \cdot \ldots \cdot\left(Q_{l-1}\left[z_{\infty}\right] \Gamma_{\rho}^{*}\right) Q_{l}\left[z_{\infty}\right] \Omega,
$$

and we set $\varphi_{k, k-1}:=\Omega$.

Step 1: There is a constant $C<\infty$ depending on $\xi, \rho$ and $\sum_{n} \gamma_{n}$ such that, for all $\overline{k, l \in \mathbb{N}}$ with $k \leq l$

$$
\left\|\varphi_{k, l}-\varphi_{k, l-1}\right\| \leq C \gamma_{l} .
$$

By definition of $\varphi_{k, l}$ and since $\Omega=\Gamma_{\rho}^{*} \chi_{\rho} \Omega$

$$
\varphi_{k, l}-\varphi_{k, l-1}=\prod_{n=k}^{l-1}\left(Q_{n}\left[z_{\infty}\right] \Gamma_{\rho}^{*}\right)\left(Q_{l}\left[z_{\infty}\right]-\chi_{\rho}\right) \Omega,
$$

where the empty product in the case $k=l$ is to interpret as the identity operator. Since on $U_{n},\left\|Q_{n} \Gamma_{\rho}^{*}\right\|=\left\|Q_{n}\right\| \leq\left\|Q_{n}-\chi_{\rho}\right\|+1 \leq \exp \left\|Q_{n}-\chi_{\rho}\right\|$ it follows that

$$
\left\|\varphi_{k, l}-\varphi_{k, l-1}\right\| \leq \exp \left(\sum_{n=k}^{l-1}\left\|Q_{n}\left[z_{\infty}\right]-\chi_{\rho}\right\|\right)\left\|Q_{l}\left[z_{\infty}\right]-\chi_{\rho}\right\|,
$$


and hence it remains to estimate $\left\|Q_{n}\left[z_{\infty}\right]-\chi_{\rho}\right\|$. By definition of $Q_{n}$, on $U_{n}$,

$$
Q_{n}-\chi_{\rho}=-\bar{\chi}_{\rho}\left(H_{\bar{\chi}_{\rho}}^{(n)}\right)^{-1} \bar{\chi}_{\rho}\left(H^{(n)}-H_{0,0}^{(n)}\right) \chi_{\rho}
$$

and by estimates in the proof of Lemma 15,

$$
\left\|\left(H_{\bar{\chi}_{\rho}}^{(n)}\right)^{-1} \bar{\chi}_{\rho}\right\| \leq \frac{8}{\rho} \frac{1}{1-\xi}, \quad\left\|H^{(n)}-H_{0,0}^{(n)}\right\| \leq \xi \gamma_{n} .
$$

Equation (60), combined with the estimates (59), and (61) prove Step 1 with

$$
C:=\frac{8}{\rho} \frac{\xi}{1-\xi} \exp \left(\frac{8}{\rho} \frac{\xi}{1-\xi} \sum_{n \geq 0} \gamma_{n}\right) \text {. }
$$

Step 2: For all $k \in \mathbb{N}$, the limit

$$
\varphi_{k, \infty}:=\lim _{n \rightarrow \infty} \varphi_{k, n}
$$

exists, the convergence being uniform in $s$, and $\varphi_{k, \infty} \neq 0$ for $k$ sufficiently large.

Summing up the estimates from Step 1 for all $l$ with $l \geq n+1$ we arrive at

$$
\left\|\varphi_{k, \infty}-\varphi_{k, n}\right\| \leq C \sum_{l=n+1}^{\infty} \gamma_{l} \rightarrow 0, \quad n \rightarrow \infty,
$$

uniformly in $s$. Specializing this inequality to $n=k-1$ so that $\varphi_{k, n}=\varphi_{k, k-1}=\Omega$, we see that $\left\|\varphi_{k, \infty}-\Omega\right\|<1=\|\Omega\|$ and hence $\varphi_{k, \infty} \neq 0$ for sufficiently large $k$.

Step 3: For all $k \in \mathbb{N}$,

$$
H^{(k)}\left[z_{\infty}\right] \varphi_{k, \infty}\left[z_{\infty}\right]=0, \quad \text { and } \quad \varphi_{k, \infty}\left[z_{\infty}\right] \neq 0 .
$$

Since $H^{(k)}\left[z_{\infty}\right]$ is a bounded operator and by (58),

$$
\begin{aligned}
H^{(k)}\left[z_{\infty}\right] \varphi_{k, \infty} & =\lim _{n \rightarrow \infty} H^{(k)}\left[z_{\infty}\right] \varphi_{k, n} \\
& =\lim _{n \rightarrow \infty}\left(\rho \Gamma_{\rho}^{*} \chi_{1}\right)^{n-k+1} H^{(n+1)}\left[z_{\infty}\right] \Omega .
\end{aligned}
$$

Using $H^{(n+1)}\left[z_{\infty}\right] \Omega=E^{(n+1)}\left(z_{\infty}\right) \Omega+\left(H^{(n+1)}\left[z_{\infty}\right]-H_{0,0}^{(n+1)}\left[z_{\infty}\right]\right) \Omega$ and

$$
\left|E^{(n+1)}\left(z_{\infty}\right)\right| \leq \frac{\rho}{2}, \quad\left\|H^{(n+1)}\left[z_{\infty}\right]-H_{0,0}^{(n+1)}\left[z_{\infty}\right]\right\| \leq \gamma_{n} \leq \gamma_{0}
$$

we see that the limit (62) vanishes because $\lim _{n \rightarrow \infty} \rho^{n}=0$.

From $\varphi_{k-1, n}=\left(Q_{k-1}\left[z_{\infty}\right] \Gamma_{\rho}^{*}\right) \varphi_{k, n}$, the boundedness of the operator $Q_{k}$, and from Step 2 it follows that,

$$
\varphi_{k-1, \infty}=\left(Q_{k-1}\left[z_{\infty}\right] \Gamma_{\rho}^{*}\right) \varphi_{k, \infty} .
$$

Since $\varphi_{k, \infty}$ belongs to the kernel of $H^{(k)}\left[z_{\infty}\right]$, as we have just seen, it follows from Theorem 7 that $\varphi_{k-1, \infty} \neq 0$ whenever $\varphi_{k, \infty} \neq 0$. Iterating this argument starting with $k$ so large that, by Step $2, \varphi_{k, \infty} \neq 0$, we conclude that $\varphi_{k, \infty} \neq 0$ for all $k \in \mathbb{N}$. 


\section{Analyticity of eigenvalues and eigenvectors}

This section is devoted to the proof of Theorem 1. It is essential for this proof, that a neighborhoods $V_{0} \subset V$ of $s_{0}$ and a bound $g_{1}$ on $g$ can be determined in such a way that the renormalization analysis of Sections 8 and 9 , and in particular the choices of $\rho$ and $\xi$ are independent of $s \in V_{0}$ and $g<g_{1}$. Once $V_{0}$ and $g$ are found, the assertions of Theorem 1 are derived from Proposition 17 and the uniform bounds of Sections 8 and 9 .

Proof of Theorem 1 . Let $\mu>0$ and $\mathcal{U} \subset \mathbb{C}^{\nu+1}$ be given by Hypothesis $\mathrm{I}$ and Hypothesis III, respectively. For the renormalization procedure to work, we first choose $\rho \in(0,4 / 5)$ and a open neighborhood $V_{0} \subset V$ of $s_{0}$, both small enough, so that $C_{\gamma} \rho^{\mu}<1$ and

$$
\overline{B\left(E_{\text {at }}(s), \rho\right)} \subset\{z \mid(s, z) \in \mathcal{U}\}, \quad \text { if } \quad s \in V_{0} .
$$

This is possible since $s \mapsto E_{\text {at }}(s)$ is continuous. Let $\xi=\sqrt{\rho} /\left(4 C_{\chi}\right)$. Next we pick small positive constants $\alpha_{0}, \beta_{0}$, and $\gamma_{0}$ such that

$$
\alpha_{0}<\frac{\rho}{2}, \quad \beta_{0} \leq \frac{\rho}{8 C_{\chi}}, \quad \gamma_{0} \leq \frac{\rho}{8 C_{\chi}},
$$

and in addition

$$
\beta_{0}+\frac{C_{\beta} / \rho}{1-\left(C_{\chi} \rho^{\mu}\right)^{2}} \gamma_{0}^{2} \leq \frac{\rho}{8 C_{\chi}} .
$$

By Theorems 13 and 23, there exists a $g_{1}>0$ such that for $|g| \leq g_{1}$

$$
H_{g}^{(0)}[s, z]-\left(E_{\mathrm{at}}(s)-z\right) \in \mathcal{B}\left(\alpha_{0}, \beta_{0}, \gamma_{0}\right), \quad \text { for } \quad(s, z) \in \mathcal{U},
$$

where $H_{g}^{(0)}[s, z]$ is analytic on $\mathcal{U}$. We define

$$
\begin{aligned}
& \mathcal{U}_{0}:=\mathcal{U} \\
& \mathcal{U}_{n}:=\left\{(s, z) \in \mathcal{U}_{n-1}:\left|E^{n-1}(s, z)\right| \leq \rho / 8\right\},
\end{aligned}
$$

and

$$
U_{n}(s):=\left\{z \mid(s, z) \in \mathcal{U}_{n}\right\}, \quad n \in \mathbb{N} .
$$

Then, by (64), (65), and (63) the assumptions of Lemma 19 are satisfied for $s \in V_{0}$ and $U_{0}=U_{0}(s)$. It follows that, for all $n \in \mathbb{N}, H^{(n)}[s, z]=\mathcal{R}^{n} H^{(0)}[s, z]$ is welldefined for $(s, z) \in \mathcal{U}_{n}$, and that $U_{n}(s) \neq \emptyset$. By Proposition $17, H^{(n)}[s, z]$ is analytic in $\mathcal{U}_{n}^{\circ}$.

Step 1: $z_{\infty}(s)=\lim _{n \rightarrow \infty} z_{n}(s)$ exists and is analytic on $V_{0}$.

Since $H^{(n)}[s, z]$ is analytic on $\mathcal{U}_{n}^{\circ}$, so is $E^{(n)}(s, z)$. Let $z_{n}(s)$ denote the unique zero of the function $z \mapsto E_{n}(s, z)$ on $U_{n}(s)$ as determined by Lemma 19. That is,

$$
E^{(n)}\left(s, z_{n}(s)\right)=0 \text {. }
$$

By the implicit function theorem $z_{n}(s)$ is analytic in $s$. The application of the implicit function theorem is justified since $z \mapsto E^{(n)}(s, z)$ is bijective in a neighborhood of $z_{n}(s)$, and thus in this neighborhood $\partial_{z} E^{(n)}(s, z) \neq 0$. By Lemma 19 (b), 
$z_{n}(s)$ converges to $z_{\infty}(s)$ uniformly in $s \in V_{0}$. This implies the analyticity of $z_{\infty}(s)$ on $V_{0}$, by the Weierstrass approximation theorem of complex analysis.

Step 2: For $s \in V_{0}$, there exists an eigenvector $\psi(s)$ of $H(s)$ with eigenvalue $z_{\infty}(s)$, such that $\psi(s)$ depends analytically on $s$.

Since $H^{(n)}[s, z]$ is analytic on $\mathcal{U}_{n}^{\circ}$, it follows, by Proposition 17 , that

$$
Q_{n}[s, z]=\chi_{\rho}(s)-\bar{\chi}_{\rho}(s) H_{\bar{\chi}_{\rho}}^{(n)}[s, z]^{-1} \bar{\chi}_{\rho}(s) W^{(n)}[s, z] \chi_{\rho}(s)
$$

is analytic on $\mathcal{U}_{n}^{\circ}$, where $W^{(n)}:=H^{(n)}-H_{0,0}^{(n)}$. Hence, by Step $1, s \mapsto Q_{n}\left[s, z_{\infty}(s)\right]$ is analytic on $V_{0}$. It follows that

$$
\varphi_{0, n}(s):=Q_{0}\left[s, z_{\infty}(s)\right] \Gamma_{\rho}^{*} Q_{1}\left[s, z_{\infty}(s)\right] \ldots \Gamma_{\rho}^{*} Q_{n}\left[s, z_{\infty}(s)\right] \Omega
$$

is analytic on $V_{0}$. From Theorem 22 we know that these vectors converge uniformly on $V_{0}$ to a vector $\varphi^{(0)}(s) \neq 0$ and that $H^{(0)}\left[s, z_{\infty}(s)\right] \varphi^{(0)}(s)=0$. Hence $\varphi^{(0)}(s)$ is analytic on $V_{0}$ and, by the Feshbach property (Proposition 10 (ii)), the vector

$$
\psi(s)=Q_{\chi}\left[s, z_{\infty}(s)\right]\left(\varphi_{\text {at }}(s) \otimes \varphi^{(0)}(s)\right)
$$

is an eigenvector of $H(s)$ with eigenvalue with $z_{\infty}(s)$. Since $\varphi_{\text {at }}$ is analytic on $V_{0}$ we conclude that $\psi$ is analytic on $V_{0}$ as well.

Step 3: For $s \in V_{0} \cap \mathbb{R}^{\nu}, z_{\infty}(s)=\inf \sigma(H(s))$.

Let $s \in V_{0} \cap \mathbb{R}^{\nu}$. Then $H(s)$ is self-adjoint and its spectrum is a half line $[E(s), \infty)$. By Step $2, z_{\infty}(s) \geq E(s)$. We use Proposition 21 to show that $z_{\infty}(s)>$ $E(s)$ is impossible. Clearly $E_{\text {at }}(s) \in \mathbb{R}$, and $H^{(0)}[s, z]^{*}=H^{(0)}[s, \bar{z}]$ for $z \in$ $B\left(E_{\text {at }}(s), \rho\right)$ is a direct consequence of the definition of $H^{(0)}$ and the self-adjointness of $H(s)$. Hence there exists a number $a(s)<z_{\infty}(s)$ such that $H^{(0)}[s, x]$ has a bounded inverse for all $x \in\left(a(s), z_{\infty}(s)\right)$. It follows, by Theorem 7 , that $(a(s)$, $\left.z_{\infty}(s)\right) \cap \sigma(H(s))=\emptyset$. Therefore $z_{\infty}(s)=E(s)$.

\section{Appendix A. Neighborhood of effective Hamiltonians}

The purpose of this section is to prove the following theorem.

Theorem 23. Let Hypotheses I, II, and III hold for some $\mu>0$ and $\mathcal{U} \subset \mathbb{C} \times \mathbb{C}$. For every $\xi \in(0,1)$ and every triple of positive constants $\alpha_{0}, \beta_{0}, \gamma_{0}$, there exists a positive constant $g_{1}$ such that for all $g \in\left[0, g_{1}\right)$ and all $(s, z) \in \mathcal{U},\left(H_{g}(s)-\right.$ $\left.z, H_{0}(s)-z\right)$ is a Feshbach pair for $\chi(s)$, and

$$
H_{g}^{(0)}[s, z]-\left(E_{\text {at }}(s)-z\right) \in \mathcal{B}\left(\alpha_{0}, \beta_{0}, \gamma_{0}\right) .
$$

By Theorem 13 we know that we can choose $g$ sufficiently small such that the Feshbach property is satisfied. To prove (66) we explicitly compute the sequence 
of kernels $w=\left(w_{m, n}\right) \in \mathcal{W}_{\xi}$ such that $H_{g}^{(0)}[s, z]=H(w)$. To this end we recall that, by (18) and (19),

$$
H_{g}^{(0)}[s, z]=\left(E_{\mathrm{at}}-z\right)+H_{f}+\left\langle\chi_{1}\left(g W-g W \bar{\chi}\left(H_{g}-z\right)_{\bar{\chi}}^{-1} \bar{\chi} g W\right) \chi_{1}\right\rangle_{\mathrm{at}},
$$

and we expand the resolvent $\left(H_{g}-z\right)_{\bar{\chi}}^{-1}$ in a Neumann series. We find that

$$
\left\langle\chi_{1}\left(g W-g W \bar{\chi}\left(H_{g}-z\right)_{\bar{\chi}}^{-1} \bar{\chi} g W\right) \chi_{1}\right\rangle_{\text {at }}=\sum_{L=1}^{\infty}(-1)^{L-1} g^{L}\left\langle\chi_{1}(W F)^{L-1} W \chi_{1}\right\rangle_{\text {at }},
$$

where $F=\bar{\chi}\left(H_{0}-z\right)^{-1} \bar{\chi}$ is a function of $H_{f}$, that is, $F=F\left(H_{f}\right)$ with

$$
F(r):=\frac{\bar{\chi}^{2}(s, r)}{H_{\mathrm{at}}(s)-z+r},
$$

and $\bar{\chi}(s, r)=\bar{P}_{\text {at }}(s) \otimes 1+P_{\text {at }}(s) \otimes \bar{\chi}_{1}(r)$. Since $W=a(G)+a^{*}(G)$, the $L$ th term in this series is a sum of $2^{L}$ terms. We label them by $L$-tuples $\sigma=\left(\sigma_{1}, \sigma_{2}, \ldots, \sigma_{L}\right)$, with $\sigma_{i} \in\{-,+\}$, and we set $a^{+}(G):=a^{*}(G), a^{-}(G):=a(G)$. With these notations

$$
\left\langle\chi_{1}(W F)^{L-1} W \chi_{1}\right\rangle_{\mathrm{at}}=\sum_{\underline{\sigma} \in\{-,+\}^{L}}\left\langle\chi_{1} \prod_{j=1}^{L-1}\left\{a^{\sigma_{j}}(G) F\left(H_{f}\right)\right\} a^{\sigma_{L}}(G) \chi_{1}\right\rangle_{\text {at }} .
$$

Next we use a variant of Wick's theorem (see [6]) to expand each term of the sum (69) in a sum of normal ordered terms. Explicitly, this means that in each term of (69) the pull through formulas

$$
f\left(H_{f}\right) a^{*}(k)=a^{*}(k) f\left(H_{f}+|k|\right), \quad a(k) f\left(H_{f}\right)=f\left(H_{f}+|k|\right) a(k),
$$

and the canonical commutation relations are used to move all creation operators to the very left, and all annihilation operators to the right of all other operators. To write down the result we introduce the multi-indices

$$
\underline{m, p, n, q}:=\left(m_{1}, p_{1}, n_{1}, q_{1}, \ldots, m_{L}, p_{L}, n_{L}, q_{L}\right) \in\{0,1\}^{4 L},
$$

which run over the sets $I_{L}:=\left\{m, p, n, q \in\{0,1\}^{4 L} \mid m_{l}+p_{l}+n_{l}+q_{l}=1\right\}$. The numbers $m_{l}, p_{l}, n_{l}, q_{l}$ may be thought of as flags that indicate the position of the operator $a^{\sigma_{l}}(k)$ in a given normal-ordered term: $m_{l}=1\left(n_{l}=1\right)$ if it is a noncontracted creation (annihilation) operator, $p_{l}=1\left(q_{l}=1\right)$ if it is a contracted creation (annihilation) operator. We obtain

$$
\begin{aligned}
& \left\langle\chi_{1}(W F)^{L-1} W \chi_{1}\right\rangle_{\mathrm{at}} \\
& =\sum_{\underline{m, p, n, q} \in I_{L}} \int d k_{\underline{m}} d \tilde{k}_{\underline{n}}\left\{\prod_{l=1}^{L} a^{*}\left(k_{m_{l}}\right)^{m_{l}}\right\} V_{\underline{m, p, n, q}}\left(H_{f}, k_{\underline{m}}, \tilde{k}_{\underline{n}}\right)\left\{\prod_{l=1}^{L} a\left(\tilde{k}_{n_{l}}\right)^{n_{l}}\right\},
\end{aligned}
$$


with

$$
\begin{aligned}
& V_{\underline{m, p, n, q}}\left(r, k_{\underline{m}}, \tilde{k}_{\underline{n}}\right)=\chi_{1}\left(r+r_{0}(\underline{m}, \underline{n})\right) \\
& \quad \times\left\langle\left\{\prod_{l=1}^{L-1} G\left(k_{m_{l}}\right)^{m_{l}} G^{*}\left(\tilde{k}_{n_{l}}\right)^{n_{l}} a^{*}(G)^{p_{l}} a(G)^{q_{l}} F\left(H_{f}+r+r_{l}(\underline{m}, \underline{n})\right)\right\}\right. \\
& \left.\quad \times G\left(k_{m_{L}}\right)^{m_{L}} G^{*}\left(\tilde{k}_{n_{L}}\right)^{n_{L}} a^{*}(G)^{p_{L}} a(G)^{q_{l}}\right\rangle_{\mathrm{at}, \Omega} \chi_{1}\left(r+r_{L}(\underline{m}, \underline{n})\right),
\end{aligned}
$$

where $\langle A\rangle_{\text {at }, \Omega}:=\left(\Omega,\langle A\rangle_{\text {at }} \Omega\right), \Omega \in \mathcal{F}$ being the vacuum vector. Moreover

$$
\begin{array}{lll}
k_{\underline{m}}:=\left(m_{1} k_{1}, \ldots, m_{L} k_{L}\right), & d \tilde{k}_{\underline{m}}:=\prod_{l=1, m_{l}=1}^{L} d k_{l} \\
\tilde{k}_{\underline{\underline{n}}}:=\left(n_{1} \tilde{k}_{1}, \ldots, n_{L} \tilde{k}_{L}\right), & d \tilde{k}_{\underline{\underline{n}}}:=\prod_{l=1, n_{l}=1}^{L} d \tilde{k}_{l}
\end{array}
$$

and

$$
r_{l}(\underline{m}, \underline{n})=\sum_{\substack{i \leq l \\ m_{l}=1}}\left|k_{i}\right|+\sum_{\substack{i \geq l+1 \\ n_{l}=1}}\left|\tilde{k}_{i}\right| .
$$

Upon summing (70) for $L=1$ through $\infty$ we collect all terms with equal numbers $M=|\underline{m}|:=\sum_{l=1}^{L} m_{l}$ and $N=|\underline{n}|:=\sum_{l=1}^{L} n_{l}$ of creation and annihilation operators, respectively. To this end we need to relabel the integration variables. That is, we distribute the $M+N$ variables $k_{1}, \ldots, k_{M} \in \mathbb{R}^{3} \times\{1,2\}$ and $\tilde{k}_{1}, \ldots, \tilde{k}_{N} \in \mathbb{R}^{3} \times\{1,2\}$ into the $M+N$ arguments of $V_{m, p, n, q}(r, \cdot, \cdot)$ designated by $m_{l}=1$ and $n_{l}=1$. Explicitly this is done by

$$
\sigma_{\underline{m}}\left(k_{1}, \ldots, k_{M}\right)=\left(m_{1} k_{\underline{m}(1)}, \ldots, m_{L} k_{\underline{m}(L)}\right), \quad \underline{m}(l)=\sum_{j=1}^{l} m_{j} .
$$

We obtain

$$
\begin{aligned}
\sum_{L \geq 1}(-1)^{L-1} g^{L}\left\langle\chi_{1}(W F)^{L-1} W \chi_{1}\right\rangle_{\text {at }} & \\
& =\sum_{M+N \geq 1} \int_{B^{M+N}} a^{*}\left(k^{(M)}\right) \hat{w}_{M, N}\left(H_{f}, K\right) a\left(\tilde{k}^{(N)}\right) d K
\end{aligned}
$$

where

$$
\begin{array}{r}
\hat{w}_{M, N}(r, K) \\
=\sum_{L \geq M+N}(-1)^{L-1} g^{L} \sum_{\substack{m, p, n, q \in I_{L} \\
|\underline{m}|=M,|\underline{m}|=N}} V_{\underline{m, p, n, q}}\left(r, \sigma_{\underline{m}}\left(k^{(M)}\right), \sigma_{\underline{n}}\left(\tilde{k}^{(N)}\right)\right)
\end{array}
$$


and $K=\left(k^{(M)}, \tilde{k}^{(N)}\right)$. Hence $H_{g}^{(0)}=H(w)$ with

$$
w_{0,0}(r)=E_{a t}-z+r+\sum_{L \geq 1}(-1)^{L-1} g^{L} \sum_{\substack{p, q \in\{0,1\}^{2 L} \\ p_{l}+q_{l}=1}} V_{\underline{0, p, 0, q}}(r),
$$

and $w_{M, N}(r, K)$ given by the symmetrisation of $\hat{w}_{M, N}(r, K)$ with respect to $k_{1}, \ldots, k_{M} \in \mathbb{R}^{3}$ and $\tilde{k}_{1}, \ldots, \tilde{k}_{N} \in \mathbb{R}^{3}$, respectively.

It remains to show that $H(w)-\left(E_{\text {at }}-z\right)$ belongs to the ball $\mathcal{B}\left(\alpha_{0}, \beta_{0}, \gamma_{0}\right)$ for $g$ sufficiently small. To this end we need the following estimates on the operatorvalued function (68) and on its derivative,

$$
F^{\prime}(r)=-\frac{\bar{\chi}^{2}(s, r)}{\left(H_{\mathrm{at}}(s)-z+r\right)^{2}}+\frac{P_{\mathrm{at}}(s) \otimes 2 \bar{\chi}_{1}(s, r) \partial_{r} \bar{\chi}_{1}(s, r)}{H_{\mathrm{at}}(s)-z+r} .
$$

Lemma 24. Let Hypothesis I and III hold for some $\mu>0$ and $\mathcal{U}$. Then

$$
\begin{aligned}
& C_{0}:=\sup _{(s, z) \in \mathcal{U}} \sup _{r \geq 0}\left\|\left(H_{f}+1\right) F\left(H_{f}+r\right)\right\|<\infty \\
& C_{1}:=\sup _{(s, z) \in \mathcal{U}} \sup _{r \geq 0}\left\|\left(H_{f}+1\right) F^{\prime}\left(H_{f}+r\right)\right\|<\infty,
\end{aligned}
$$

for $F$ given by (68).

Proof. To show that $C_{0}$ is finite we estimate

$$
\begin{aligned}
\sup _{r \geq 0} & \left\|\left(H_{f}+1\right) \frac{\bar{\chi}^{2}\left(s, H_{f}+r\right)}{H_{\mathrm{at}}(s)-z+H_{f}+r}\right\| \\
= & \sup _{r, q \geq 0}\left\|(q+1) \frac{\bar{P}_{\mathrm{at}}(s) \otimes 1+P_{\mathrm{at}}(s) \otimes \bar{\chi}_{1}^{2}(r+q)}{H_{\mathrm{at}}(s)-z+q+r}\right\| \\
\leq & \sup _{r, q \geq 0}\left\|(q+1) \frac{\bar{P}_{\mathrm{at}}(s)}{H_{\mathrm{at}}(s)-z+q+r}\right\| \\
& \quad+\sup _{r, q \geq 0}\left\|(q+1) \frac{\bar{\chi}_{1}^{2}(r+q)}{E_{\mathrm{at}}(s)-z+q+r}\right\|\left\|P_{\mathrm{at}}(s)\right\| .
\end{aligned}
$$

By Hypothesis III, both terms are bounded on $\mathcal{U}$. Similarly $C_{1}$ is estimated using (74).

Proof of Theorem 23. Let Hypothesis I and III hold for some $\mu>0$ and $\mathcal{U}$. Let $0<\xi<1$. By Theorem 13 we know that there exists a $g_{0}>0$ such that for all $|g|<g_{0},\left(H_{g}-z, H_{0}-z\right)$ on $\mathcal{U}$ is a Feshbach pair for $\chi$. Let $(s, z) \in \mathcal{U}$. First we derive upper bounds for $V_{\underline{m, p, n, q}}$ and $\partial_{r} V_{\underline{m, p, n, q}}$. Inserting $\left(H_{f}+1\right)^{-1}\left(H_{f}+1\right)$ in front of $F\left(H_{f}+r+r_{l}(\underline{m}, \underline{\underline{n}})\right)$ we obtain, from Lemma 24 , that

$$
\begin{aligned}
& \left|V_{\underline{m, p, n, q}}\left(r, k_{\underline{m}}, \tilde{k}_{\underline{n}}\right)\right| \\
& \leq\left\{\prod_{l=1}^{L}\left\|G\left(k_{m_{l}}\right)\right\|^{m_{l}}\left\|G\left(k_{n_{l}}\right)\right\|^{n_{l}}\|G\|_{\omega}^{p_{l}+q_{l}}\right\} C_{0}^{L-1} \sup _{s:(s, z) \in \mathcal{U}}\left\|P_{\text {at }}(s)\right\| .
\end{aligned}
$$


Let $C_{\text {at }}:=\sup _{s:(s, z) \in \mathcal{U}}\left\|P_{\text {at }}(s)\right\|$. Similarly, using (71), (74) and (75) we estimate

$$
\begin{aligned}
\left|\partial_{r} V_{\underline{m, p, n, q}}\left(r, k_{\underline{m}}, \tilde{k}_{\underline{n}}\right)\right| \\
\leq 2\left\|\chi_{1}^{\prime}\right\|_{\infty} \cdot\left\{\prod_{l=1}^{L}\left\|G\left(k_{m_{l}}\right)\right\|^{m_{l}}\left\|G\left(k_{n_{l}}\right)\right\|^{n_{l}}\|G\|_{\omega}^{p_{l}+q_{l}}\right\} C_{0}^{L-1} C_{\mathrm{at}} \\
\quad+\sum_{j=1}^{L-1} \mid\left\langle\left\{\prod_{l=1}^{j-1} G\left(k_{m_{l}}\right)^{m_{l}} G^{*}\left(\tilde{k}_{n_{l}}\right)^{n_{l}} a^{*}\left(G_{g}\right)^{p_{l}} a(G)^{q_{l}} F\left(H_{f}+r+r_{l}(\underline{m}, \underline{n})\right)\right\}\right. \\
\quad \times G\left(k_{m_{j}}\right)^{m_{j}} G^{*}\left(\tilde{k}_{n_{j}}\right)^{n_{j}} a^{*}(G)^{p_{j}} a(G)^{q_{j}} F^{\prime}\left(H_{f}+r+r_{j}(\underline{m}, \underline{n})\right) \\
\quad \times\left\{\prod_{l=j+1}^{L-1} G\left(k_{m_{l}}\right)^{m_{l}} G^{*}\left(\tilde{k}_{n_{l}}\right)^{n_{l}} a^{*}(G)^{p_{l}} a(G)^{q_{l}} F\left(H_{f}+r+r_{l}(\underline{m}, \underline{n})\right)\right\} \\
\left.\quad \times G\left(k_{m_{L}}\right)^{m_{L}} G^{*}\left(\tilde{k}_{n_{L}}\right)^{n_{L}} a^{*}(G)^{p_{L}} a\left(G_{g}\right)^{q_{l}}\right\rangle_{\mathrm{at}, \Omega} \mid \\
\leq\left\{\prod_{l=1}^{L}\left\|G\left(k_{m_{l}}\right)\right\|^{m_{l}}\left\|G\left(k_{n_{l}}\right)\right\|^{n_{l}}\|G\|_{\omega}^{p_{l}+q_{l}}\right\} C_{\mathrm{at}} C_{0}^{L-2}\left(2\left\|_{1}^{\prime}\right\|_{\infty} C_{0}+(L-1) C_{1}\right) .
\end{aligned}
$$

With the help of (75) and (77) we can now prove the theorem. From (73) and (75) it follows that

$$
\begin{aligned}
\left|w_{0,0}(0)-\left(E_{\text {at }}-z\right)\right| & \leq \sum_{L=2}^{\infty} g^{L} \sum_{\substack{\frac{p, q}{p_{l}+q_{l}=1}\\
}}\left|V_{0, p, 0, q}(0)\right| \\
& \leq \sum_{L=2}^{\infty} g^{L} \sum_{\substack{p, q \in\{0,1\}^{2 L} \\
p_{l}+q_{l}=1}}\|G\|_{\omega}^{L} C_{0}^{L-1} C_{\text {at }} \\
& \leq C_{\text {at }} \sum_{L=2}^{\infty} 2^{L} g^{L}\|G\|_{\omega}^{L} C_{0}^{L-1},
\end{aligned}
$$

which can be made smaller than any positive $\alpha_{0}$ for small $g$. Estimate (77) implies that

$$
\begin{aligned}
\left\|w_{0,0}^{\prime}-1\right\|_{\infty} & =\sup _{r}\left|w_{0,0}^{\prime}[r]-1\right| \\
& \leq \sup _{r} \sum_{L=2}^{\infty} g^{L} \sum_{\substack{p, q \in\{0,1\}^{2 L} \\
p_{l}+q_{l}=1}}\left|\partial_{r} V_{0, p, 0, q}(r)\right|
\end{aligned}
$$




$$
\begin{aligned}
& \leq \sum_{L=2}^{\infty} g^{L} \sum_{\substack{p, q \in\{0,1\}^{2 L} \\
p_{l}+q_{l}=1}}\|G\|_{\omega}^{L} C_{\mathrm{at}} C_{0}^{L-2}\left(2 C_{0}\left\|\chi_{1}^{\prime}\right\|_{\infty}+(L-1) C_{1}\right) \\
& \leq \sum_{L=2}^{\infty} g^{L}\|G\|_{\omega}^{L} 2^{L} C_{\mathrm{at}} C_{0}^{L-2}\left(2 C_{0}\left\|\chi_{1}^{\prime}\right\|_{\infty}+(L-1) C_{1}\right),
\end{aligned}
$$

which can be made smaller than any positive $\beta_{0}$ for small $g$. It remains to show that $\left\|\left(w_{M, N}\right)_{M+N \geq 1}\right\|_{\mu, \xi} \leq \gamma_{0}$ for $g$ sufficiently small. By (72)

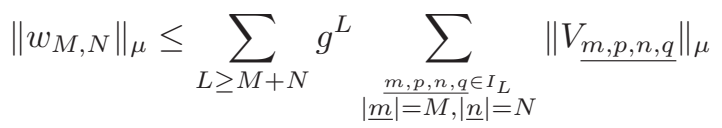

where, by a triangle inequality and by (75) and (77)

$$
\begin{aligned}
\left\|V_{\underline{m, p, n, q}}\right\|_{\mu} \leq & \left(\int_{B^{M+N}}\left\|V_{\underline{m, p, n, q}}(K)\right\|_{\infty}^{2} \frac{d K}{|K|^{2+2 \mu}}\right)^{1 / 2} \\
& +\left(\int_{B^{M+N}}\left\|\partial_{r} V_{\underline{m, p, n, q}}(K)\right\|_{\infty}^{2} \frac{d K}{|K|^{2+2 \mu}}\right)^{1 / 2} \\
\leq & \|G\|_{\mu}^{M+N}\|G\|_{\omega}^{L-(M+N)} S_{L}
\end{aligned}
$$

with $S_{L}:=C_{\mathrm{at}} C_{0}^{L-2}\left(C_{0}+2\left\|\chi_{1}^{\prime}\right\|_{\infty} C_{0}+(L-1) C_{1}\right)$, and

$$
\|G\|_{\mu}:=\left(\int_{\mathbb{R}^{3}}\|G(k)\|^{2} \frac{d k}{|k|^{2+2 \mu}}\right)^{1 / 2} .
$$

Combining (78) and (79) and find

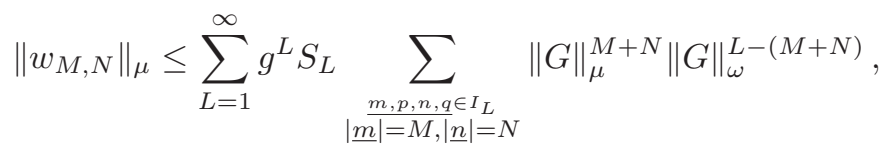

where the condition $L \geq M+N$ has been relaxed to $L \geq 1$. Therefore

$$
\begin{aligned}
\| & \left(w_{M, N}\right)_{M+N \geq 1} \|_{\mu, \xi} \\
& =\sum_{M+N \geq 1} \xi^{-(M+N)}\left\|w_{N, M}\right\|_{\mu} \\
& \leq \sum_{L=1}^{\infty} g^{L} S_{L}\|G\|_{\omega}^{L} \sum_{M+N \geq 1} \xi^{-(M+N)} \sum_{\substack{\frac{m, p, n, q \in I_{L}}{|m|=M,|\underline{n}|=N} \\
\mid \underline{m}}}\left(\|G\|_{\omega}^{-1}\|G\|_{\mu}\right)^{M+N} \\
& \leq \sum_{L=1}^{\infty} g^{L} S_{L}\|G\|_{\omega}^{L}\left(\sum_{\underline{m, p, n, q} \in I_{1}}\left(\xi^{-1}\|G\|_{\omega}^{-1}\|G\|_{\mu}\right)^{m_{1}+n_{1}}\right)^{L} \\
& \leq \sum_{L=1}^{\infty} g^{L} S_{L}\|G\|_{\omega}^{L}\left(2+2 \xi^{-1}\|G\|_{\omega}^{-1}\|G\|_{\mu}\right)^{L} .
\end{aligned}
$$


This can be made smaller than any positive $\gamma_{0}$ for small coupling $g$. It follows that we can find a $g_{1}>0$ such that on $\mathcal{U},(66)$ holds for all $g \in\left[0, g_{1}\right)$. This concludes the proof.

\section{Appendix B. Technical auxiliaries}

Let $L^{2}\left(\mathbb{R}^{3} \times\{1,2\}, \mathcal{L}\left(\mathcal{H}_{\text {at }}\right)\right)$ be the Banach space of (weakly) measurable functions $T: \mathbb{R}^{3} \times\{1,2\} \rightarrow \mathcal{L}\left(\mathcal{H}_{\text {at }}\right)$ with $\int\|T(k)\|^{2} d k<\infty$, and let

$$
\|T\|_{\omega}:=\left(\int\|T(k)\|^{2}\left(|k|^{-1}+1\right) d k\right)^{1 / 2} .
$$

Lemma 25. If $T \in L^{2}\left(\mathbb{R}^{3} \times\{1,2\}, \mathcal{L}\left(\mathcal{H}_{\text {at }}\right)\right)$, then

$$
\begin{aligned}
\left\|a(T)\left(H_{f}+1\right)^{-1 / 2}\right\| & \leq\left(\int\|T(k)\|^{2}|k|^{-1} d k\right)^{1 / 2}, \\
\left\|a^{*}(T)\left(H_{f}+1\right)^{-1 / 2}\right\| & \leq\|T\|_{\omega} .
\end{aligned}
$$

For a proof of this lemma see, e.g., [5].

Lemma 26. Suppose the function $F: U \rightarrow \mathcal{L}\left(\mathcal{H}_{\mathrm{at}}, L^{2}\left(\mathbb{R}^{3} ; \mathcal{H}_{\mathrm{at}}\right)\right), s \mapsto F_{s}$ is uniformly bounded and suppose for a.e. $k \in \mathbb{R}^{3}$ and all $s \in U$, there exists an operator $F_{s}(k) \in \mathcal{L}\left(\mathcal{H}_{\text {at }}\right)$ such that $F_{s}(k) \varphi=\left(F_{s} \varphi\right)(k)$ for all $\varphi \in \mathcal{H}_{\text {at }}$. If for a.e. $k \in \mathbb{R}^{3}$, the function $s \mapsto F_{s}(k) \in \mathcal{L}\left(H_{\mathrm{at}}\right)$ is analytic, then $F$ is analytic.

Proof. Let $h \in L^{2}\left(\mathbb{R}^{3}\right)$ and $\varphi_{1}, \varphi_{2} \in \mathcal{H}_{\text {at }}$, and suppose $\gamma$ is a nullhomotopic closed curve in $U$. Then

$$
\begin{aligned}
\int_{\gamma}\left(h \otimes \varphi_{1}, F_{s} \varphi_{2}\right) d s & =\int_{\gamma} \int \bar{h}(k)\left(\varphi_{1}, F_{s}(k) \varphi_{2}\right) d k d s \\
& =\int \bar{h}(k) \int_{\gamma}\left(\varphi_{1}, F_{s}(k) \varphi_{2}\right) d s d k=0,
\end{aligned}
$$

where we interchanged the order of integration, which is justified since $F$ is uniformly bounded. It follows that $s \mapsto\left(h \otimes \varphi_{1}, F_{s} \varphi_{2}\right)$ is analytic. By linearity we conclude that $s \mapsto\left(\psi, F_{s} \varphi_{2}\right)$ is analytic for all $\psi$ in a dense linear subset of $\mathcal{H}_{\text {at }} \otimes \mathfrak{h}$. This and the uniform boundedness imply strong analyticity, see for example the remark following Theorem 3.12 of Chapter III in [16].

Proposition 27. Let $R \ni s \mapsto T(s)$ be an analytic family. Suppose there exists an isolated non-degenerate eigenvector $E(s)$ with analytic projection operator $P(s)$. Let $\bar{P}(s):=1-P(s)$ and let

$$
\begin{gathered}
\Gamma:=\{(s, z) \in R \times \mathbb{C} \mid(T(s)-z) \text { is a bijection from } D(T(s)) \cap \operatorname{Ran} \bar{P}(s) \\
\text { to } \operatorname{Ran} \bar{P}(s) \text { with bounded inverse }\} .
\end{gathered}
$$

Then $\Gamma$ is open and $(s, z) \mapsto(T(s)-z)^{-1} \bar{P}(s)$ is analytic on $\Gamma$. 
Proof. Let $\left(s_{0}, z_{0}\right) \in \Gamma$. There exists in a neighborhood of $s_{0}$ a bijective operator $U(s): \mathcal{H} \rightarrow \mathcal{H}$, analytic in $s$, such that $U(s) P(s) U(s)^{-1}=P\left(s_{0}\right)$ and hence $U(s) \bar{P}(s) U(s)^{-1}=\bar{P}\left(s_{0}\right)$ ( [20] Thm. XII.12). The operator $\widetilde{T}(s)=$ $U(s) T(s) U(s)^{-1}$ is an analytic family. It leaves $\operatorname{Ran} \bar{P}\left(s_{0}\right)$ invariant and thus $\widetilde{T}(s)\left\lceil\operatorname{Ran} \bar{P}\left(s_{0}\right): \operatorname{Ran} \bar{P}\left(s_{0}\right) \cap \mathcal{D}(\widetilde{T}(s)) \rightarrow \operatorname{Ran} \bar{P}\left(s_{0}\right)\right.$ is an analytic family as well. By this and the fact that $\left(\widetilde{T}\left(s_{0}\right)-z_{0}\right)\left\lceil\operatorname{Ran} \bar{P}\left(s_{0}\right)\right.$ is bijective with bounded inverse since $\left(s_{0}, z_{0}\right) \in \Gamma$, it follows by [20] Thm. XII.7 that in a neighborhood of $\left(s_{0}, z_{0}\right)$, $(\widetilde{T}(s)-z)\left\lceil\operatorname{Ran} \bar{P}\left(s_{0}\right)\right.$ is bijective with bounded inverse and $(\widetilde{T}(s)-z)^{-1} \bar{P}\left(s_{0}\right)$ is analytic in both variables. Thus in this neighborhood also the function $(T(s)-z)\lceil\bar{P}(s)$ is bijective with bounded inverse and $(T(s)-z)^{-1} \bar{P}(s)$ is an analytic function of two variables.

Theorem 28. Suppose the assumptions of Lemma 19 hold. Then in the norm of $\mathcal{L}\left(\mathcal{H}_{\text {red }}\right)$,

$$
\lim _{n \rightarrow \infty} H^{(n)}\left(z_{\infty}\right)=\lambda H_{f}
$$

for some $\lambda \in \mathbb{C}$.

Proof. We recall the notations $H^{(n)}\left(z_{\infty}\right)=H\left(w^{(n)}\left(z_{\infty}\right)\right)$ and $E^{(n)}\left(z_{\infty}\right)=$ $w_{0,0}^{(n)}\left(z_{\infty}, 0\right)$. Using the decomposition

$$
\begin{aligned}
H^{(n)}\left(z_{\infty}\right)= & \left(H^{(n)}\left(z_{\infty}\right)-w_{0,0}^{(n)}\left(z_{\infty}, H_{f}\right)\right) \\
& +\left(w_{0,0}^{(n)}\left(z_{\infty}, H_{f}\right)-E^{(n)}\left(z_{\infty}\right)\right)+E^{(n)}\left(z_{\infty}\right),
\end{aligned}
$$

the theorem will follow from Steps 1 and 2 below.

Step 1: $\lim _{n \rightarrow \infty}\left\|H^{(n)}\left(z_{\infty}\right)-w_{0,0}^{(n)}\left(z_{\infty}, H_{f}\right)\right\|=0$ and $\lim _{n \rightarrow \infty} E^{(n)}\left(z_{\infty}\right)=0$.

From Lemma 18 we know that

$$
H^{(n)}(z)-\rho^{-1} E^{(n-1)}(z) \in \mathcal{B}\left(\alpha_{n}, \beta_{n}, \gamma_{n}\right),
$$

for $z \in U_{n}$. By (34) this implies that

$$
\left\|H^{(n)}\left(z_{\infty}\right)-w_{0,0}^{(n)}\left(z_{\infty}, H_{f}\right)\right\| \leq\left\|w^{(n)}\left(z_{\infty}\right)-w_{0,0}^{(n)}\left(z_{\infty}\right)\right\|_{\mu, \xi} \leq \gamma_{n} \rightarrow 0 \quad(n \rightarrow \infty) .
$$

By (80),

$$
\left|E^{(n)}(z)\right| \leq \rho \alpha_{n+1}+\rho\left|E^{(n+1)}(z)\right|, \quad z \in U_{n} .
$$

Iterating (81), we find

$$
\left|E^{(n)}(z)\right| \leq \sum_{k=1}^{m} \rho^{k} \alpha_{n+k}+\rho^{m}\left|E^{(n+m)}(z)\right|,
$$

which yields,

$$
\left|E^{(n)}\left(z_{n+m}\right)\right| \leq \sum_{k=1}^{\infty} \rho^{k} \alpha_{n+k}
$$


Since $E^{(n)}$ is continuous and $\lim _{n \rightarrow \infty} z_{n}=z_{\infty}$, we arrive at

$$
\left|E^{(n)}\left(z_{\infty}\right)\right| \leq \sum_{k=1}^{\infty} \rho^{k} \alpha_{n+k} \rightarrow 0, \quad(n \rightarrow \infty) .
$$

Step 2: There exists a $\lambda \in \mathbb{C}$ such that

$$
\lim _{n \rightarrow \infty}\left(w_{0,0}^{(n)}\left(z_{\infty}, r\right)-w^{(n)}\left(z_{\infty}, 0\right)\right)=\lambda r,
$$

uniformly in $0 \leq r \leq 1$.

To abbreviate the notation, we set $T^{(n)}\left(z_{\infty}, r\right):=w_{0,0}^{(n)}\left(z_{\infty}, r\right)-w^{(n)}\left(z_{\infty}, 0\right)$. From [3] (3.105-3.107), we have

$$
T^{(n)}\left(z_{\infty}, r\right)=\rho^{-1} T^{(n-1)}\left(z_{\infty}, \rho r\right)+e^{(n-1)}\left(z_{\infty}, r\right),
$$

with $e^{(n-1)}\left(z_{\infty}, 0\right)=0$ and

$$
\sup _{r \in[0,1]}\left(\left|\partial_{r} e^{(n)}\left(z_{\infty}, r\right)\right|+\left|e^{(n)}\left(z_{\infty}, r\right)\right|\right) \leq C \gamma_{n}^{2} .
$$

Iterating (82), we arrive at

$$
T^{(n)}\left(z_{\infty}, r\right)=\rho^{-n} T^{(0)}\left(z_{\infty}, \rho^{n} r\right)+\sum_{k=0}^{n-1} \rho^{-(n-1-k)} e^{(k)}\left(z_{\infty}, \rho^{n-1-k} r\right) .
$$

To prove Step 2 we now show that, uniformly in $r \in[0,1]$,

$$
\lim _{n \rightarrow \infty} T^{(n)}\left(z_{\infty}, r\right)=r\left(\partial_{r} T^{(0)}\left(z_{\infty}, 0\right)+\sum_{k=0}^{\infty} \partial_{r} e^{(k)}\left(z_{\infty}, 0\right)\right) .
$$

Note that the series on the right hand side converges by (83). Given $\epsilon>0$ we choose $K$ so large that

$$
\sum_{k=K}^{\infty} C \gamma_{k}^{2} \leq \epsilon
$$

By $(84)$ and the triangle inequality, we find for $n \geq K$, (suppressing $\left.z_{\infty}\right)$

$$
\begin{aligned}
& \left|T^{(n)}(r)-r\left(\partial_{r} T^{(0)}(0)-\sum_{k=0}^{\infty} \partial_{r} e^{(k)}(0)\right)\right| \\
& \leq\left|\rho^{-n} T^{(0)}\left(\rho^{n} r\right)-r \partial_{r} T^{(0)}(0)\right|+\sum_{k=0}^{K}\left|\rho^{-(n-1-k)} e^{(k)}\left(\rho^{n-1-k} r\right)-r \partial_{r} e^{(k)}(0)\right| \\
& \quad+\sum_{k=K+1}^{\infty}\left|\rho^{-(n-1-k)} e^{(k)}\left(\rho^{n-1-k} r\right)\right|+\sum_{k=K+1}^{\infty}\left|r \partial_{r} e^{(k)}(0)\right| .
\end{aligned}
$$

The first two terms on the right hand side converge to zero as $n$ tends to infinity because $T^{(n)}(0)=0$ and $e^{(k)}(0)=0$. The last term on the right hand side is bounded by $\epsilon$, which follows from Eqns. (83) and (85). Using again (83) and (85) 
we see that the first term on the last line is bounded by $\epsilon$ as well, since, by the mean value theorem, $\alpha^{-1}\left|e^{(n)}(\alpha r)\right| \leq \sup _{\xi \in[0,1]}\left|e^{(n)^{\prime}}(\alpha \xi)\right| r$ for $\alpha, r \in[0,1]$.

\section{Acknowledgements}

We thank Jürg Fröhlich and Israel Michael Sigal for numerous discussions on the renormalization technique. M. Griesemer also thanks Volker Bach for explaining the results of [4], and Ira Herbst for the hospitality at the University of Virginia, where large parts of this work were done.

\section{References}

[1] J.E. Avron and A. Elgart, Adiabatic theorem without a gap condition: Two-level system coupled to quantized radiation field, Phys. Rev. A 58 (6) (1998), 4300-4306.

[2] J. E. Avron and A. Elgart, Adiabatic theorem without a gap condition, Comm. Math. Phys. 203 (2) (1999), 445-463.

[3] V. Bach, T. Chen, J. Fröhlich and I. M. Sigal, Smooth Feshbach map and operatortheoretic renormalization group methods, J. Funct. Anal. 203 (1) (2003), 44-92.

[4] V. Bach, J. Fröhlich and A. Pizzo, Infrared-finite algorithms in QED: The groundstate of an atom interacting with the quantized radiation field, Comm. Math. Phys. 264 (1) (2006), 145-165.

[5] V. Bach, J. Fröhlich and I. M. Sigal, Quantum electrodynamics of confined nonrelativistic particles, Adv. Math. 137 (2) (1998), 299-395.

[6] V. Bach, J. Fröhlich and I. M. Sigal, Renormalization group analysis of spectral problems in quantum field theory, Adv. Math. 137 (2) (1998), 205-298.

[7] T. Chen, Infrared renormalization in non-relativistic QED and scaling criticality, J. Funct. Anal. 254 (10) (2008), 2555-2647.

[8] C. Cohen-Tannoudji, J. Dupont-Roc and G. Grynberg, Photons and atoms: Introduction to quantum electrodynamics, John Wiley \& Sons, 1989.

[9] J. Fröhlich, On the infrared problem in a model of scalar electrons and massless, scalar bosons, Ann. Inst. H. Poincaré Sect. A (N.S.) 19 (1973), 1-103.

[10] J. Fröhlich, M. Griesemer and I. M. Sigal, Spectral renormalization group, arXiv:0811.2616.

[11] M. Griesemer, Non-relativistic matter and quantized radiation, In J. Derezinski and H. Siedentop, editors, Large Coulomb Systems, volume 695 of Lect. Notes Phys., pages 217-248. Springer, 2006.

[12] M. Griesemer and D. Hasler, On the smooth Feshbach-Schur map, J. Funct. Anal. 254 (9) (2008), 2329-2335.

[13] M. Griesemer, E.H. Lieb and M. Loss, Ground states in non-relativistic quantum electrodynamics, Invent. Math. 145 (3) (2001), 557-595.

[14] D. Hasler and I. Herbst, Absence of ground states for a class of translation invariant models of non-relativistic QED, Comm. Math. Phys. 279 (3) (2008), 769-787.

[15] W. Hunziker, Distortion analyticity and molecular resonance curves, Ann. Inst. H. Poincaré Phys. Théor. 45 (4) (1986), 339-358. 
[16] T. Kato, Perturbation theory for linear operators, Classics in Mathematics. SpringerVerlag, Berlin, 1995. Reprint of the 1980 edition.

[17] S. G. Krantz, Function theory of several complex variables, The Wadsworth \& Brooks/Cole Mathematics Series. Wadsworth \& Brooks/Cole Advanced Books \& Software, Pacific Grove, CA, second edition, 1992.

[18] E. H. Lieb and M. Loss, Existence of atoms and molecules in non-relativistic quantum electrodynamics, Adv. Theor. Math. Phys. 7 (4) (2003), 667-710.

[19] A. Pizzo, One-particle (improper) states in Nelson's massless model, Ann. Henri Poincaré 4 (3) (2003), 439-486.

[20] M. Reed and B. Simon, Methods of modern mathematical physics. IV. Analysis of operators, Academic Press [Harcourt Brace Jovanovich Publishers], New York, 1978.

[21] S. Teufel, Effective N-body dynamics for the massless Nelson model and adiabatic decoupling without spectral gap, Ann. Henri Poincaré 3 (5) (2002), 939-965.

[22] S. Teufel, A note on the adiabatic theorem without gap condition, Lett. Math. Phys. 58 (3) (2002), 261-266 (2002).

Marcel Griesemer

Fachbereich Mathematik

Universität Stuttgart

D-70569 Stuttgart

Germany

e-mail: marcel@mathematik.uni-stuttgart.de

David G. Hasler

Department of Mathematics

College of William \& Mary

Williamsburg, VA 23187-8795

USA

e-mail: dghasler@wm.edu

Communicated by Claude Alain Pillet.

Submitted: November 24, 2008.

Accepted: March 4, 2009. 